\title{
A Preliminary Study of the Burgers Equation with Symbolic Computation
}

\author{
Russell G. Derickson* and Roger A. Pielke, Sr. $\dagger$ \\ ${ }^{*}$ R. G. Derickson and Associates, 224 Cypress Circle, Broomfield, Colorado 80020; $\dagger$ Department \\ of Atmospheric Sciences, Colorado State University, Fort Collins, Colorado 80521 \\ E-mail: rgderick@aol.com, pielke@hercules.atmos.colostate.edu
}

Received October 4, 1999; revised May 2, 2000

\begin{abstract}
A novel approach based on recursive symbolic computation is introduced for the approximate analytic solution of the Burgers equation. Once obtained, appropriate numerical values can be inserted into the symbolic solution to explore parametric variations. The solution is valid for both inviscid and viscous cases, covering the range of Reynolds number from 500 to infinity, whereas current direct numerical simulation (DNS) methods are limited to Reynolds numbers no greater than 4000 . What further distinguishes the symbolic approach from numerical and traditional analytic techniques is the ability to reveal and examine direct nonlinear interactions between waves, including the interplay between inertia and viscosity. Thus, preliminary efforts suggest that symbolic computation may be quite effective in unveiling the "anatomy" of the myriad interactions that underlie turbulent behavior. However, due to the tendency of nonlinear symbolic operations to produce combinatorial explosion, future efforts will require the development of improved filtering processes to select and eliminate computations leading to negligible high order terms. Indeed, the initial symbolic computations present the character of turbulence as a problem in combinatorics. At present, results are limited in time evolution, but reveal the beginnings of the well-known "saw tooth" waveform that occurs in the inviscid case (i.e., $\operatorname{Re}=\infty$ ). Future efforts will explore more fully developed 1-D flows and investigate the potential to extend symbolic computations to 2-D and 3-D. Potential applications include the development of improved subgrid scale (SGS) parameterizations for large eddy simulation (LES) models, and studies that complement DNS in exploring fundamental aspects of turbulent flow behavior. (c) 2000 Academic Press

Key Words: turbulence; nonlinear; symbolic computation.
\end{abstract}

\section{INTRODUCTION}

The Burgers equation, first presented by Bateman [2] and named after Burgers [5, 6], has been explored throughout the years to test numerical algorithms and to explore 1-D 
turbulence, often referred to as "burgulence." Indeed, fundamental knowledge about the nature of certain turbulent processes (e.g., Burgers [5, 6], Lighthill [18], Blackstock [3]) has been gleaned from the Burgers equation. Despite its fundamental nonlinearity, closed-form analytical solutions have been obtained for the Burgers equation for a wide range of initial and boundary conditions (e.g., Whitham [22], Hopf [17], Cole [9], Fletcher [13, 14]). These analytical solutions serve as benchmarks for numerical solutions, but also provide insights in their own right. Several current studies of turbulence in which the Burgers equation plays a dominant role underscore its ongoing critical relevancy within the scientific community (e.g., Gotoh and Kraichnan [15], Chen and Kraichnan [8], E et al. [11], Gurbatov et al. [16], Bouchaud et al. [4], Avellaneda et al. [1], and Chekhlov and Yakhot [7]).

The essence of turbulence is embodied in the quadratic, nonlinear convection terms of the general, 3-D Navier-Stokes (N-S) equations. Turbulence is a fully three-dimensional phenomenon, and, as such, can be understood completely only with a 3-D view. However, the Burgers equation, although 1-D, possesses a fundamental quadratic nonlinearity and is viewed as an appropriate starting "model" for studying turbulence. In fact Fletcher [13] describes how the 1-D Burgers equation is suitable not only to explore and validate numerical models but also serves as a reasonable means to study such physical processes as shock waves, acoustic transmission, traffic flow, turbulent flow in a channel, compressible flow turbulence, supersonic flow around airfoils, wave propagation in a thermo-elastic medium, and the dynamics of bubbles in a liquid.

In this paper, a new approach developed by one of the authors (Derickson [10]) is presented in which symbolic computation is employed to obtain approximate analytical solutions to the Burgers equations. Through a recursive process, symbolic representations of momentum are obtained continuously in space at discrete increments in time. Once obtained, appropriate numerical values can be inserted into the symbolic solution to explore parametric variations. The solution is valid for both viscous and inviscid cases, covering the range of Reynolds number from 500 to infinity. Unlike numerical approaches, high $\mathrm{Re}$ flows present no difficulty to the symbolic method and, in fact, require less computational effort than solutions at low Re. This is both ironic and fortuitous because high Re flows are least understood and most in need of further study. Similarly, the symbolic method induces no aliasing or false dispersion of waves, because the solution is analytic and continuous in space. To the best of the authors' knowledge, symbolic computation, although it has been used widely in other areas (e.g., Scott et al. [21]), has never been undertaken in the study of fluid turbulence.

A particular distinguishing feature of the symbolic approach is its ability to reveal the direct nonlinear interactions between waves and the interplay between inertia and viscosity at all spatial scales, including the small, dissipation scales. Such an approach finds relevancy in the study of the basic fluid mechanics of turbulence and potentially could enhance the use of direct numerical simulation (DNS) in exploring fundamental turbulent processes in the inertial sub-range and dissipation scales. Large eddy simulation (LES) models, which are becoming the "workhorse" in both research and practical studies of geophysical and engineering flows, are in urgent need of better parameterizations of turbulence at sub-grid scales (SGS) where inertia and dissipation interact. Symbolic computation of turbulence may lead to the development of improved SGS representations.

The preliminary results presented in this paper demonstrate the potential insights gained by the symbolic computation of the Burgers equation. However, due to the prodigious number of terms generated by nonlinear interactions in the symbolic explorations, and 
limitations in computer power and memory, the initial effort was necessarily limited in scope. With accrued experience in use of the symbolic approach, and access to greater computing power, future efforts can greatly expand the preliminary results. What is now viewed as classic work by Feigenbaum [12] in the study of chaos in one-dimension (1-D), numerical computations revealed patterns and universal numbers that became the backbone of chaos theory. Analogous insights, patterns, and universal relationships in fluid turbulence potentially may be discovered by expanded use of symbolic computation. What is particularly encouraging about advances in symbolic software programs and in computer hardware since Feigenbaum's studies of chaos in the early 1970s is the potential to explore 2-D and 3-D cases.

\section{THE BURGERS EQUATION}

2.1. The viscous Burgers equation. The viscous Burgers equation for velocity $U=$ $U_{0}+u$, where $U_{0}$ is a constant and $v$ is viscosity, is

$$
\frac{\partial u}{\partial t}=-\left(U_{0}+u\right) \frac{\partial u}{\partial x}+v \frac{\partial^{2} u}{\partial x^{2}} .
$$

The equation includes the local time derivative of momentum, the nonlinear convection term (i.e., a quadratic nonlinearity), and the second order viscous diffusion term. The equation does not contain a pressure derivative term, unlike the general Navier-Stokes equations. Solutions to (1) experience a decay of momentum (hence, decay of kinetic energy) with time due to the effects of viscosity.

Casting the equation in non-dimensional form yields

$$
\frac{\partial u^{\prime}}{\partial t^{\prime}}=-\left(1+u^{\prime}\right) \frac{\partial u^{\prime}}{\partial x^{\prime}}+\operatorname{Re}^{-1} \frac{\partial^{2} u^{\prime}}{\partial x^{\prime 2}},
$$

where the various non-dimensional variables are $u^{\prime}=u / U_{0}, U_{0}^{\prime}=1, x^{\prime}=x / L_{0}, t^{\prime}=t U_{0} /$ $L_{0}$, and Reynolds number, $\operatorname{Re}=U_{0} L_{0} / v . L_{0}$ is a characteristic scale length, which corresponds to a periodic spatial domain in the current study.

2.2. The inviscid Burgers equation. The inviscid Burgers equation, in non-dimensional form, is simply

$$
\frac{\partial u^{\prime}}{\partial t^{\prime}}=-\left(1+u^{\prime}\right) \frac{\partial u^{\prime}}{\partial x^{\prime}},
$$

in which the second order viscous term in (2) has been excluded. This is equivalent to $\operatorname{Re}=\infty$. The inviscid equation contains the essential quadratic nonlinearity, but no viscous damping. Thus any solution to this equation must conserve momentum and kinetic energy with evolution in time.

2.3. Analytic solutions of the Burgers equation. Several closed-form analytic solutions have been found for the Burgers equation for a wide range of initial and boundary conditions (Whitham [22], Hopf [17], Cole [9], Fletcher [13, 14]). Fletcher [14] provided the following solution for the non-dimensional viscous Burgers equation,

$$
u(x, t)=\int_{-\infty}^{\infty} \frac{x-\xi}{t} \exp \{-0.5 \operatorname{Re} F\} d \xi / \int_{-\infty}^{\infty} \exp \{-0.5 \operatorname{Re} F\} d \xi,
$$


Exact Analytic Solution to Viscous Burgers Equation

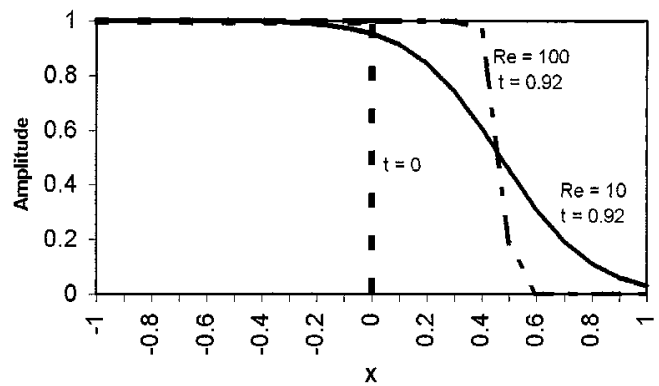

FIG. 1. Schematic of exact analytic solution of viscous Burgers equation at non-dimensional time $t=0.92$, for $\operatorname{Re}=10$ and $\operatorname{Re}=100$. Discontinuous initial condition at $t=0$ indicated by bold dashed line.

where $F(\xi ; x, t)=\int_{0}^{\xi} u_{0}\left(\xi^{\prime}\right) d \xi^{\prime}+0.5(x-\xi)^{2} / t$. The initial conditions on $u(x, t)$ are given by $u_{0}(x)=u(x, 0)=\{1$ if $-1 \leq x \leq 0$; or 0 if $0<x \leq 1$. $\}$, and the boundary conditions are $u(-1, t)=1$ and $u(1, t)=0$. The solution applies to $-1 \leq x \leq 1$ for $t \geq 0$. In (4), $u(x, t)$ represents a slightly different formulation compared to $(2)$, in that $u(x, t)$ is the total velocity. Fletcher's solution is based on a discontinuous initial condition, as displayed in Fig. 1, but a multitude of other initial conditions are possible.

A sharp wave front is maintained over time for high values of Re, but is smoothed at low Re. Such a front would also develop with a smooth initial condition such as a sinusoid in a periodic domain (e.g., Derickson [10], Fletcher [14]): the higher Re, the steeper the front. The front represents a balance between the creation of small waves through quadratic nonlinear interactions and dissipation due to viscosity. In the limit of no viscosity (i.e., $\operatorname{Re}=\infty$ ), a sharp "saw tooth" wave forms in the periodic domain.

Analytic solutions such as the preceding serve to elucidate the effect of certain parametric variations and have been used as benchmarks for numerical schemes. However, they do not unveil the details of the nonlinear interactions underlying the solution.

\section{SYMBOLIC COMPUTATION OF THE BURGERS EQUATION}

3.1. Initializing and performing the symbolic computations. Both the viscous and inviscid versions of the non-dimensional Burgers equation, (2) and (3), are computed symbolically on a periodic spatial domain with a non-dimensional length of unity (i.e., the original dimensional domain length is $L_{0}$.). A smooth sinusoidal function is specified as the initial condition, or fundamental, for the momentum,

$$
u^{\prime}\left(x^{\prime}, 0\right)=A \sin \left(\kappa^{\prime} x^{\prime}\right)
$$

which has a non-dimensional wavelength of unity, corresponding to the length of the periodic spatial domain. Unlike the analytical solution presented in Subsection 2.3, which assumes a discontinuous initial state, a smooth initial condition has been assumed here. The intent is to observe the creation of higher order harmonics and not assume them at the start.

Prior to performing the computations, it is useful to eliminate the non-dimensional constant velocity of unity in (2) and (3) to simplify computational results. Through a Galilean transformation this is equivalent to following the steep wave front generated by the quadratic 
nonlinearity of the equation and eliminates a large number of terms that would result otherwise from the symbolic operations. Thus, (2) and (3) are modified to

$$
\frac{\partial u^{\prime}}{\partial t^{\prime}}=-u^{\prime} \frac{\partial u^{\prime}}{\partial x^{\prime}}+\operatorname{Re}^{-1} \frac{\partial^{2} u^{\prime}}{\partial x^{\prime 2}}
$$

and

$$
\frac{\partial u^{\prime}}{\partial t^{\prime}}=-u^{\prime} \frac{\partial u^{\prime}}{\partial x^{\prime}}
$$

Integration of (2a) over a discrete time increment leads to the following exact, recursive expression, where the primes denoting non-dimensional variables have been dropped for convenience,

$$
u^{\tau+1}=u^{\tau}+\Delta t \overline{\left[-u \frac{\partial u}{\partial x}+\operatorname{Re}^{-1} \frac{\partial^{2} u}{\partial x^{2}}\right]} \text {. }
$$

The integration of (3a) differs only in the omission of the viscous term. In (6), $\Delta t$ is the time increment, the superscript and overbar of the bracketed terms represent the time average over the time increment, and $\tau$ corresponds to the time step, in which $\tau=0$ initially. Thus, recursive application of (6) represents an exact analytic solution at discrete time steps, $m \Delta t$, where $m$ is a positive integer.

An iterative technique was developed to approximate (6), using a trapezoidal time averaging operator, in which equal weighting is given to the new and old time steps (i.e., effectively a Crank-Nicolson implicit approach applied to the symbolic integration). Symbolic computation of the spatial terms proceeds in a straightforward manner for each iteration in a given time step. To begin each time step in the recursion, the first iteration utilizes a forward-in-time operator as

$$
u_{*}^{\tau+1}=u^{\tau}+\Delta t\left[-u \frac{\partial u}{\partial x}+\operatorname{Re}^{-1} \frac{\partial^{2} u}{\partial x^{2}}\right]^{\tau} .
$$

That is, the time average of the bracketed derivative terms in (6) is initially approximated by the symbolic representation at time level $\tau$. Then $u_{*}^{\tau+1}$, the first iterative value of $u^{\tau+1}$, is obtained from (7a), and spatial derivatives are computed for time level $\tau+1$, based on $u_{*}^{\tau+1}$. In the second iteration, the time average of the derivative terms is approximated by the arithmetic average of their symbolic representations at $\tau$ and $\tau+1$ to obtain an updated symbolic version of $u^{\tau+1}$, as

$$
u^{\tau+1}=u^{\tau}+\frac{\Delta t}{2}\left\{\left[-u \frac{\partial u}{\partial x}+\operatorname{Re}^{-1} \frac{\partial^{2} u}{\partial x^{2}}\right]^{\tau}+\left[-u_{*} \frac{\partial u_{*}}{\partial x}+\operatorname{Re}^{-1} \frac{\partial^{2} u_{*}}{\partial x^{2}}\right]^{\tau+1}\right\}
$$

Spatial derivatives at the $\tau+1$ time level then can be updated, with values at the $\tau$ level being held constant, and the iterative process can be repeated with (7b). However, sensitivity tests with multiple iterations suggest that a single trapezoidal averaging application is sufficient. That is, only one forward-in-time and one trapezoidal computation are necessary at each time step. Additional iterations primarily produce higher order harmonics of negligible magnitude rather than improve solution accuracy. The overall procedure is applied 
recursively to obtain symbolic representations for each succeeding time level, i.e., $u^{\tau+2}$, $u^{\tau+3}$, etc. The use of the iterative procedure yields a highly accurate, approximate analytic solution to the Burgers equation at the discrete time levels. Arbitrary accuracy is achieved by truncating only negligible high order terms during each time step.

As will be discussed in greater detail in Subsection 3.3, the symbolic computations produce an increasing number of terms with each successive time step. After a few time steps the number of terms becomes massive (i.e., combinatorial explosion) and it is therefore routinely necessary to select and eliminate (i.e., filter) negligible terms of higher order during symbolic operations within each time step. To ensure that eliminated terms are indeed negligible, it is necessary to establish upper numerical limits on $A$ and $\Delta t$, and a lower limit on Re, points that will be addressed in Subsection 3.5.

3.2. Overview of computational results. The symbolic solution of the Burgers equation was computed for seven discrete, non-dimensional time steps, which was sufficient to simulate the beginnings of the classic "sawtooth" waveform. It is important to emphasize that the seven non-dimensional time steps with the symbolic computations are equivalent to approximately 90 such time steps in a comparable numerical solution. This estimate is based on comparisons to numerical simulations of the Burgers equation performed by Derickson [10]. Whereas the magnitude of the time step, $\Delta t$, in numerical schemes is limited by severe restrictions on the upper bounds of the Fourier and Courant numbers, the symbolic solution, which is continuous in space, has no equivalent limitation. However, the ability to maintain solution accuracy while excluding negligible high order terms does restrict the numerical magnitude of $\Delta t$ that can be applied to the symbolic solution.

A very significant advantage of the symbolic approach over comparable numerical methods is that a numerical solution represents only a single parameter value (e.g., one value of $\mathrm{Re}$ ), whereas the symbolic solution, being analytic, represents an infinite number of parametric variations. Also, the lack of restriction on an upper limit of Re in the symbolic method adds a crucial advantage over numerical counterparts, such as DNS which is limited to $\operatorname{Re} \leq 4000$.

All symbolic computations were performed with the MAPLE symbolic engine (Redfern [20]). Computations were done in a manual, interactive mode on a 90-MHZ PC, which possessed 32 megabits of RAM. To compute seven time steps took approximately 10 minutes, most of which were consumed by manual operations, computer I/O operations, and visual assessment of results at each intermediate symbolic operation. Actual computation time is estimated to be about 2 to 4 minutes. Future efforts with more computational power and memory, and with a fully automated computational process, will greatly extend the preliminary results reported in this paper.

Table I presents the symbolic results for the 1st and 3rd time steps, showing the birth and growth of harmonics and the decay of the fundamental with time. The table also reveals the general form of the solution for each discrete time level, $m \Delta t(m=1,2,3, \ldots, M)$,

$$
u(x, m \Delta t)=\sum_{n=1}^{N} F_{n} \sin (n \kappa x)
$$

where

$$
F_{n}=\left\{\sum_{j=1}^{J} C_{n_{j}} A^{n+2 j-2} \kappa^{n+2 j-3} \Delta t^{n+2 j-3}+\sum_{j=1}^{J} D_{n_{j}} A^{n+2 j-2} \kappa^{n+2 j-1} \Delta t^{n+2 j-2} \frac{1}{\operatorname{Re}}\right\}
$$




\section{TABLE I}

\section{Results of Symbolic Computation of the Burgers Equation for 1st and 3rd Time Steps}

\section{[A] 1st Time Step}

Forward-in-time iteration

Trapezoidal iteration

$$
u(x, \Delta t)=\left\{A-A \kappa^{2} \Delta t \frac{1}{\operatorname{Re}}\right\} \sin (\kappa x)-\frac{1}{2} A^{2} \kappa \Delta t \sin (2 \kappa x)
$$

$$
\begin{aligned}
u(x, \Delta t)= & \left\{A-\frac{1}{8} A^{3} \kappa^{2} \Delta t^{2}+\left(-A \kappa^{2} \Delta t+\frac{1}{8} A^{3} \kappa^{4} \Delta t^{3}\right) \frac{1}{\operatorname{Re}}\right\} \sin (\kappa x)+\left\{-\frac{1}{2} A^{2} \kappa \Delta t+\frac{3}{2} A^{2} \kappa^{3} \Delta t^{2} \frac{1}{\operatorname{Re}}\right\} \\
& \times \sin (2 \kappa x)+\left\{\frac{3}{8} A^{3} \kappa^{2} \Delta t^{2}-\frac{3}{8} A^{3} \kappa^{4} \Delta t^{3} \frac{1}{\operatorname{Re}}\right\} \sin (3 \kappa x)-\frac{1}{8} A^{4} \kappa^{3} \Delta t^{3} \sin (4 \kappa x)
\end{aligned}
$$

[B] 3rd Time Step

Trapezoidal iteration

$$
\begin{aligned}
u(x, 3 \Delta t)= & \left\{A-\frac{9}{8} A^{3} \kappa^{2} \Delta t^{2}-\frac{3}{32} A^{5} \kappa^{4} \Delta t^{4}+\left(-3 A \kappa^{2} \Delta t+\frac{75}{8} A^{3} \kappa^{4} \Delta t^{3}+\frac{383}{32} A^{5} \kappa^{6} \Delta t^{5}\right) \frac{1}{\operatorname{Re}}\right\} \sin (\kappa x) \\
& +\left\{-\frac{3}{2} A^{2} \kappa \Delta t+4 A^{4} \kappa^{3} \Delta t^{3}-\frac{67}{128} A^{6} \kappa^{5} \Delta t^{5}+\left(\frac{27}{2} A^{2} \kappa^{3} \Delta t^{2}-54 A^{4} \kappa^{5} \Delta t^{4}-\frac{5175}{128} A^{6} \kappa^{7} \Delta t^{6}\right) \frac{1}{\operatorname{Re}}\right\} \\
& \times \sin (2 \kappa x)+\left\{\frac{27}{8} A^{3} \kappa^{2} \Delta t^{2}-\frac{801}{64} A^{5} \kappa^{4} \Delta t^{4}+\frac{261}{64} A^{7} \kappa^{6} \Delta t^{6}+\left(-\frac{417}{8} A^{3} \kappa^{4} \Delta t^{3}+\frac{14349}{64} A^{5} \kappa^{6} \Delta t^{5}\right.\right. \\
& \left.\left.+\frac{11145}{128} A^{7} \kappa^{8} \Delta t^{7}\right) \frac{1}{\operatorname{Re}}\right\} \sin (3 \kappa x)+\left\{-\frac{67}{8} A^{4} \kappa^{3} \Delta t^{3}+\frac{1151}{32} A^{6} \kappa^{5} \Delta t^{5}-\frac{553}{32} A^{8} \kappa^{7} \Delta t^{7}\right. \\
& \left.+\left(\frac{363}{2} A^{4} \kappa^{5} \Delta t^{4}-\frac{24567}{32} A^{6} \kappa^{7} \Delta t^{6}-\frac{3747}{64} A^{8} \kappa^{9} \Delta t^{8}\right) \frac{1}{\operatorname{Re}}\right\} \sin (4 \kappa x) \\
& +\left\{\frac{1365}{64} A^{5} \kappa^{4} \Delta t^{4}-\frac{6175}{64} A^{7} \kappa^{6} \Delta t^{6}+\frac{513265}{4096} A^{9} \kappa^{8} \Delta t^{8}\right. \\
& \left.+\left(-\frac{36545}{64} A^{5} \kappa^{6} \Delta t^{5}+\frac{292585}{128} A^{7} \kappa^{8} \Delta t^{7}-\frac{11108435}{4096} A^{9} \kappa^{10} \Delta t^{9}\right) \frac{1}{\operatorname{Re}}\right\} \sin (5 \kappa x) \\
& +\left\{-\frac{6855}{128} A^{6} \kappa^{5} \Delta t^{5}+\frac{22233}{128} A^{8} \kappa^{7} \Delta t^{7}+\left(\frac{209829}{128} A^{6} \kappa^{7} \Delta t^{6}-\frac{2263035}{512} A^{8} \kappa^{9} \Delta t^{8}\right) \frac{1}{\operatorname{Re}}\right\} \sin (6 \kappa x)
\end{aligned}
$$

is the amplitude of each harmonic at the discrete time level $m \Delta t ; n=1,2,3, \ldots, N$ corresponds to the fundamental and each harmonic up to the $(N-1)$ st harmonic; and the index $j=1,2,3, \ldots, J$ corresponds to the respective terms associated with the fundamental and each harmonic. The fundamental is denoted by $\sin (\kappa x)$ and the odd and even harmonics are denoted by $\sin \{(2 n) \kappa x\}$ and $\sin \{(2 n+1) \kappa x\}$, respectively. Thus, $\sin (2 \kappa x)$ corresponds to the 1 st harmonic and $\sin (3 \kappa x)$ corresponds to the 2 nd harmonic, etc. $C_{n j}$ and $D_{n j}$, all of which are rational numbers, are the respective leading coefficients for each inviscid and viscous term. For convenience of analysis, the terms are viewed as inviscid-viscous pairs, $\left(C_{n j}, D_{n j}\right)$, in which each pair of terms is denoted by its leading coefficients for expediency.

Table I shows that at each time step the leading coefficients, $C_{n j}$ and $D_{n j}$, of all existing terms change, and new terms are added to the fundamental and each harmonic through the symbolic computations. The table represents a filtered solution in which negligible higher order terms were excluded during computations. As can be observed in the table, leading coefficients can become quite large, complicating the issue of discerning negligible terms. The coefficients are rational numbers, which preserve solution accuracy. MAPLE can handle extremely large integers (500,000 digits), so large leading coefficients are not a computational problem. 
The symbolic solution represents an approximate, spatially continuous analytical solution of high order accuracy at discrete time levels. The forward-in-time iteration (FI) is included for the 1st time step in Table I to elucidate the process of solution improvement. Only the final, or trapezoidal, time iteration (TI) is shown for the 3rd time step. In general, higher order terms are affected most by the second iteration in the symbolic computations, a characteristic common to numerical iterations. Results for the inviscid Burgers equation differ from the tabulated viscous solution only in the exclusion of all terms containing Re. Symbolic computations are quicker and easier to perform for the inviscid case due to the absence of the viscous terms, which cause considerably greater combinatorial interactions. However, the inviscid case, which corresponds to infinite Re, does not induce the instabilities inherent to numerical solutions at high Re.

The symbolic solution does not produce false dispersion of waves or aliasing, nor require an upper bound on Re. These are significant issues in numerical methods. The analytic nature of the symbolic solution, which is continuous in space and discrete in time, precludes false dispersion and aliasing and any restriction on maximum allowable Re. These phenomena are known to be associated with spatial discretization in finite difference methods (e.g., Derickson [10]). Spectral methods, while producing minimal false dispersion, require dealiasing at each computational time step (e.g., Fletcher [14]). Spectral DHS methods also are restricted to Re values of no greater than 4000 .

As will be elaborated in Subsection 3.5, the viscous solution is valid only for Re above a lower limit of 500. For the solution to remain valid below this lower threshold, a large number of symbolic terms containing higher order powers of $\mathrm{Re}^{-1}$ must be retained in the symbolic computations, resulting in much greater computational expenditure. This happenstance is ironic in contrast to the difficulty numerical solutions face as Re increases from small to large magnitudes, the latter of which represent the more interesting regimes of turbulent flow, in general.

Figures 2 and 3 show the evolution of the symbolic solution for $\operatorname{Re}=500$ and $\operatorname{Re}=\infty$ (i.e., the inviscid case), respectively. The initial condition and the $3 \mathrm{rd}$ and 7 th time steps are shown. Values of $A=0.3$ and $\Delta t=0.055$, which were determined to be appropriate upper numerical limits on the amplitude factor and non-dimensional time step to maintain solution validity, are reflected in the figures. The figures reveal the decay of the fundamental and the growth of harmonics, yielding a net waveform that is tending toward the well known "sawtooth" shape produced in solutions to the Burgers equation at high Re. Figure 2, corresponding to $\mathrm{Re}=500$, shows greater damping of the fundamental and slower growth of the harmonics with time. However, the differences between the two cases become visibly discernible in Figs. 2 and 3 only at the 7th time step. As revealed in Table I and Figs. 2 and 3, the fundamental and the even harmonics have positive amplitudes and the odd harmonics have negative amplitudes. This alternation of sign is more clearly shown in Fig. 4, which displays the growth of the harmonics with each successive time step. Figure 4 also more clearly shows the greater harmonic magnitudes for $\operatorname{Re}=\infty$ compared to $\operatorname{Re}=500$.

3.3. Combinatorics of nonlinear interactions. In the symbolic solution, the quadratic nonlinear interactions at each time step correspond to symbolic multiplication between momentum, represented by (8), and its derivative, i.e., $u \frac{\partial u}{\partial x}$, in which multiplications between all possible combinations of existing harmonic pairs are performed pair by pair. Referring to Appendix A and (8) for elucidation, a single nonlinear multiplication between any two 

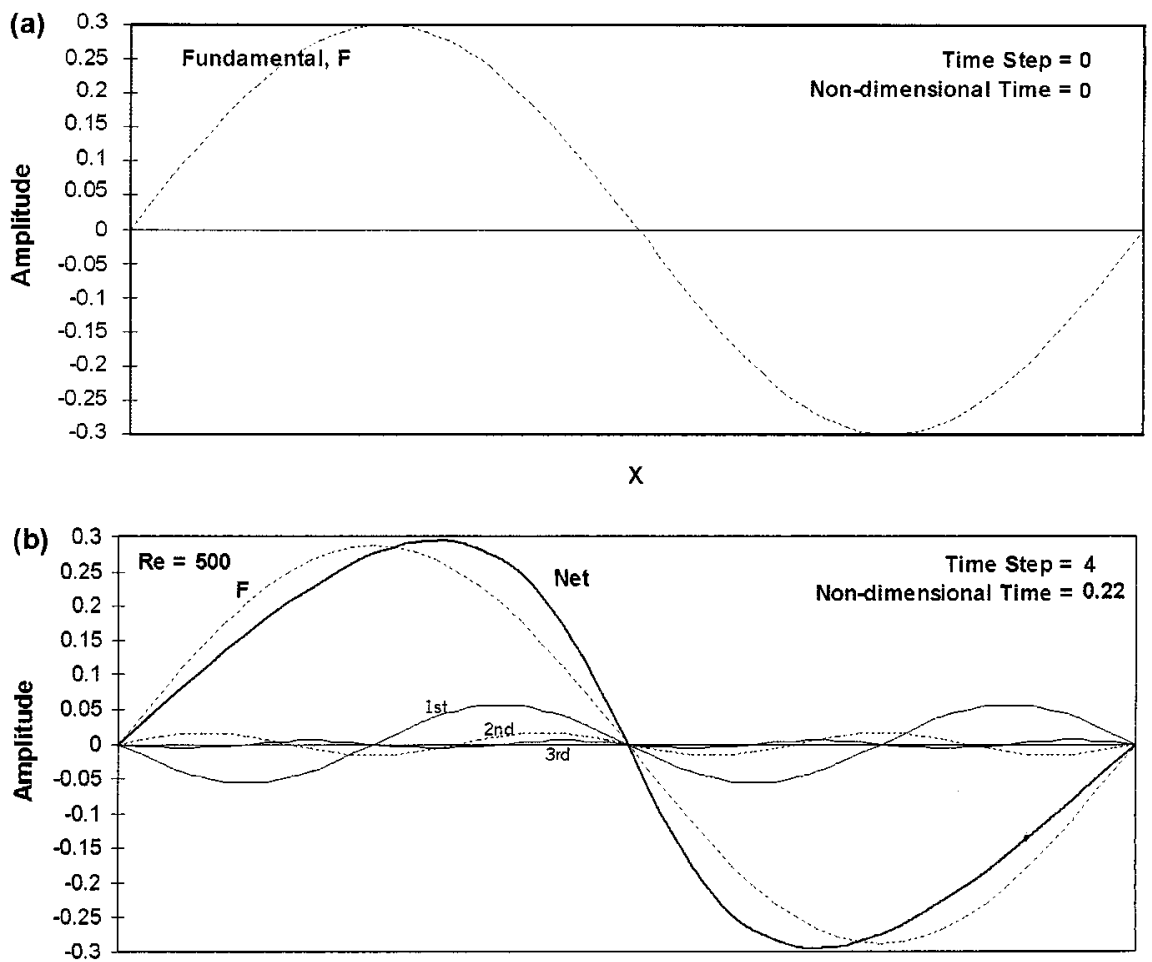

$\mathrm{x}$

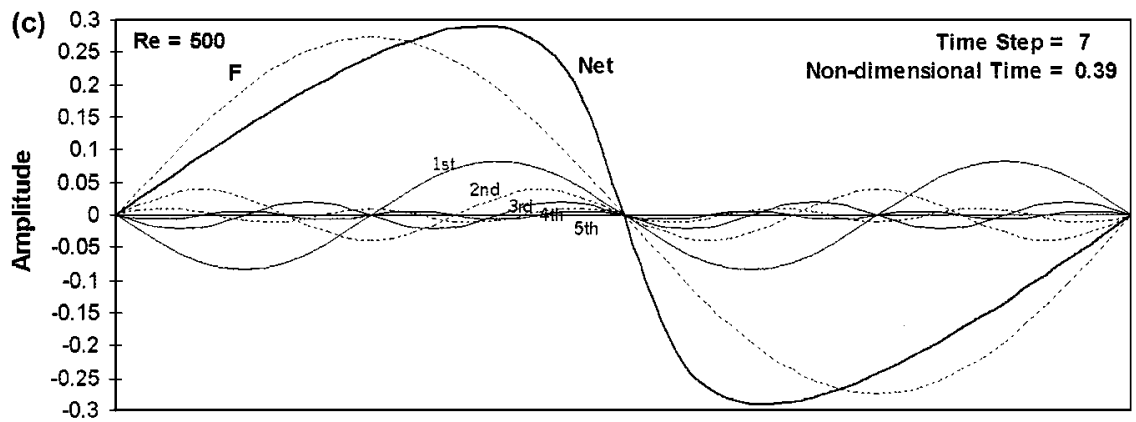

$\mathbf{x}$

FIG. 2. Time evolution of fundamental and 1st through 5 th harmonics in symbolic computation of the Burgers equation for $\operatorname{Re}=500$. Non-dimensional amplitude factor and time step are $A=0.3$ and $\Delta t=0.055$, respectively.

harmonics can be represented by the general form

$$
\begin{aligned}
& L_{c} A^{n_{1}+2 j_{1}-2} A^{n_{2}+2 j_{2}-2} \sin \left(n_{1} \kappa\right) \cos \left(n_{2} \kappa\right) \\
& \quad=\frac{1}{2} L_{c} A^{n_{1}+n_{2}+2\left(j_{1}+j_{2}-2\right)}\left[\sin \left(n_{1}+n_{2}\right) \kappa+\sin \left(n_{1}-n_{2}\right) \kappa\right],
\end{aligned}
$$

where $L_{c}$ is the leading coefficient, the ordered pair $\left(n_{1}, n_{2}\right)$ denotes the respective wavenumbers of the two interacting harmonics, and $j_{1}$ and $j_{2}$ represent the particular term associated with each harmonic, that is, its leading term, or its 2 nd or 3 rd term, etc. (refer to Table I and (8) for clarity). As explained in Appendix A, the term $\sin \left(n_{1}+n_{2}\right) \kappa$ 


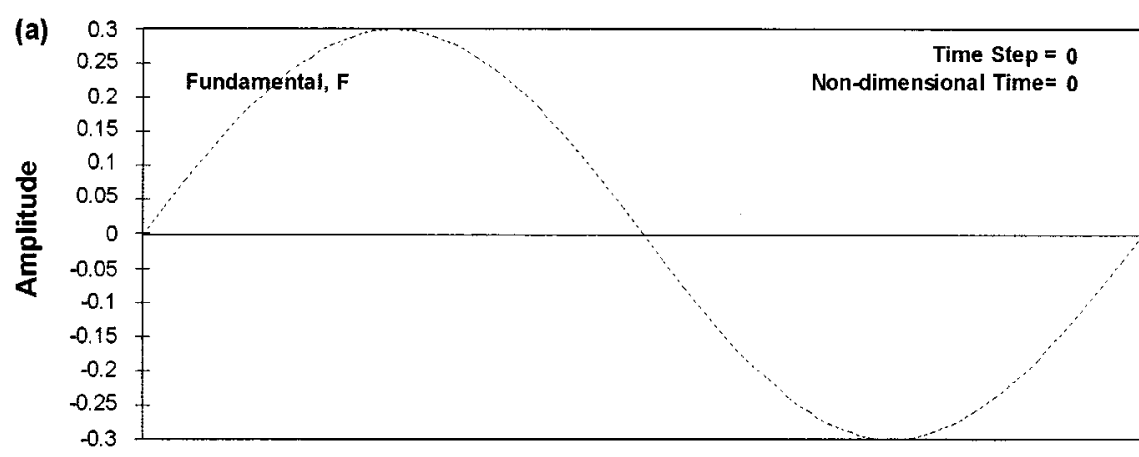

X

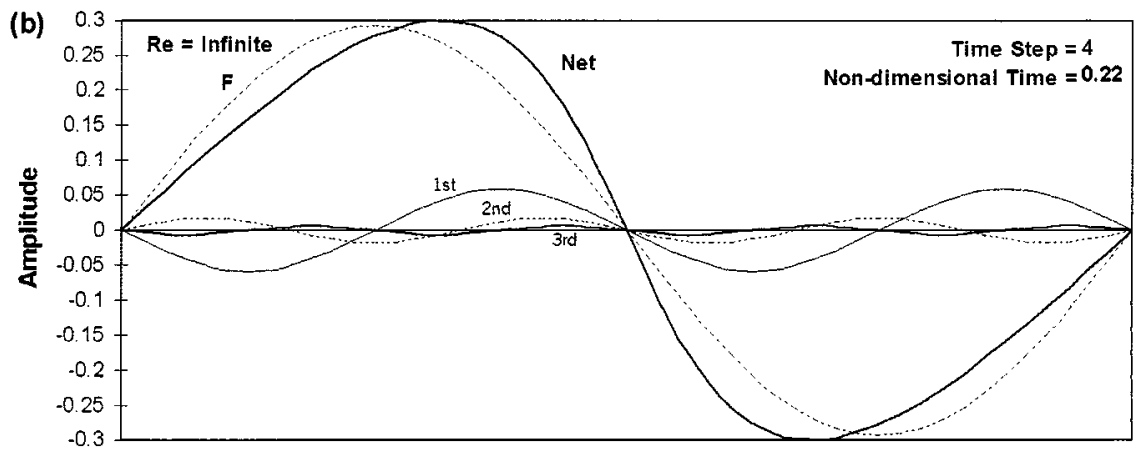

X

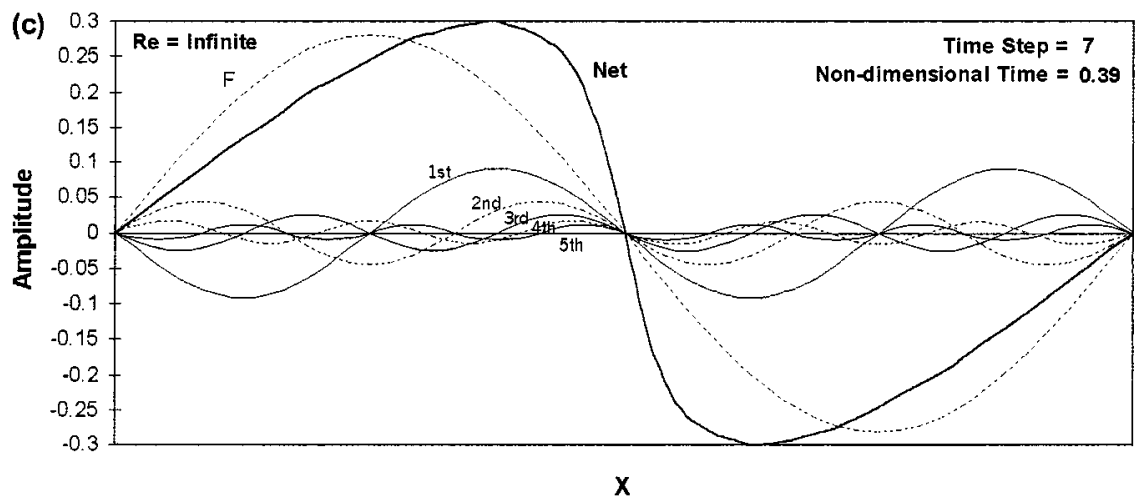

FIG. 3. Time evolution of fundamental and 1 st through 5 th harmonics in symbolic computation of the Burgers equation for infinite Re. Non-dimensional amplitude factor and time step are $A=0.3$ and $\Delta t=0.055$, respectively.

in (9) represents outscatter, or "production" of a higher order harmonic, and $\sin \left(n_{1}-n_{2}\right) \kappa$ represents backscatter to a lower order harmonic.

In general, $j_{1} \neq j_{2}$ more frequently than $j_{1}=j_{2}$. If a multiplication involves the leading term for each harmonic in the pair, then $j_{1}=j_{2}=1$. The multiplication represented by (9) may involve two inviscid terms, two viscous terms, or one inviscid and one viscous term, such that any one of the following four combinations is possible for the resulting leading coefficient, $L_{c}: L_{c}=C_{n_{1 j_{1}}} C_{n_{2 j_{2}}}, L_{c}=D_{n_{1 j_{1}}} D_{n_{2 j_{2}}}, L_{c}=C_{n_{1_{1}}} D_{n_{2 j_{2}}}$, or $L_{c}=D_{n_{1_{1}}} C_{n_{2 j_{2}}}$. The wavenumber, $\kappa$, and the time increment, $\Delta t$, have been omitted as factors in (9) because, referring to (8), their respective powers depend on whether inviscid or viscous terms, or a 
(a) $\operatorname{Re}=500$

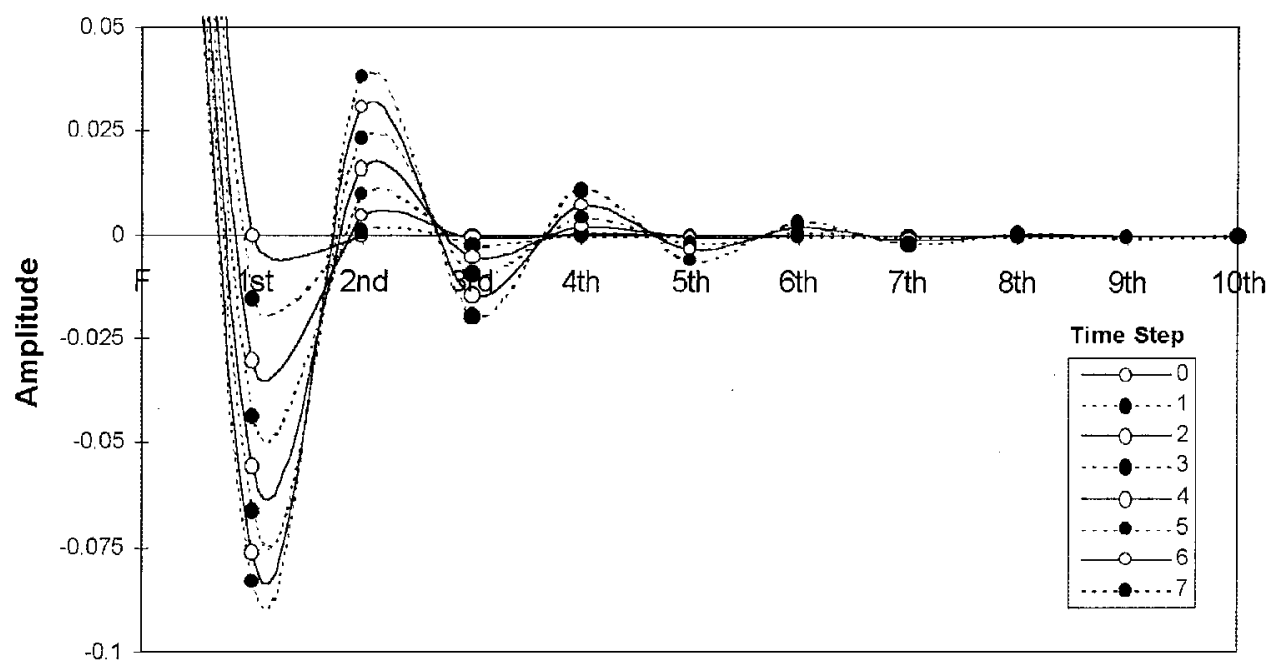

Harmonic

(b) $\mathrm{Re}=$ Infinite

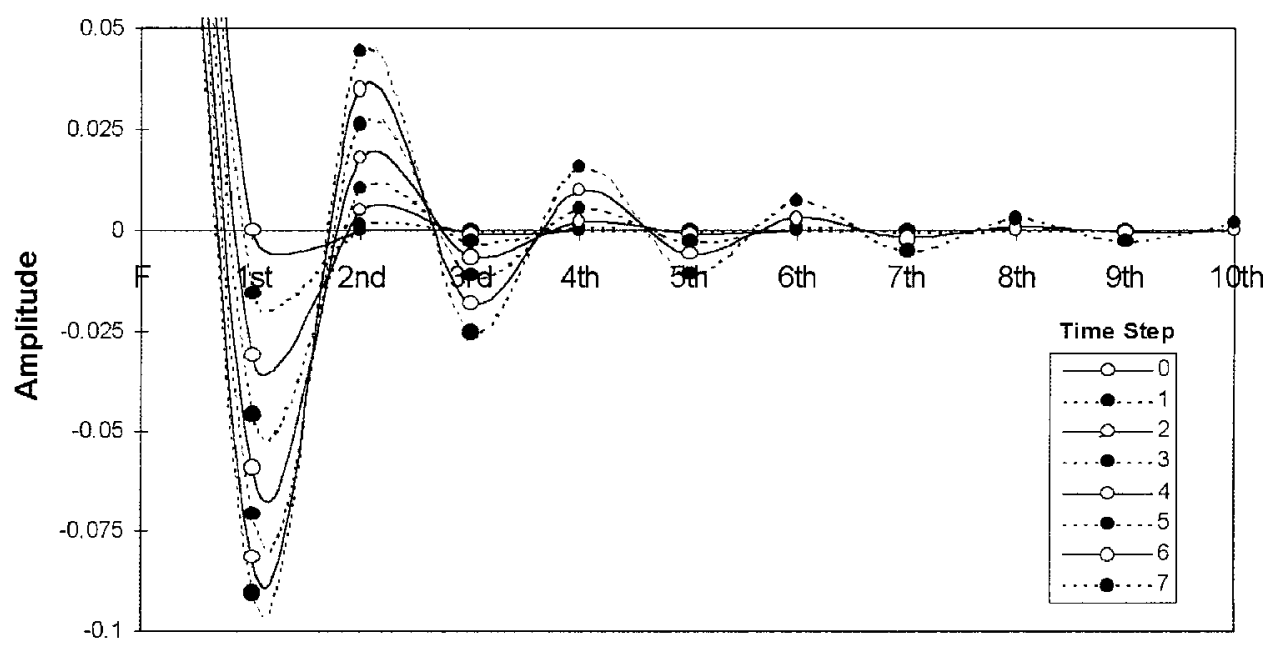

Harmonic

FIG. 4. Amplitudes of 1 st through 10th harmonics at each of seven discrete time steps in symbolic computation of the Burgers equation for (a) $\mathrm{Re}=500$ and (b) infinite Re. The artifice of continuous curve fitting between discrete harmonics helps to clarify harmonic growth in time.

mix, are involved in the multiplication. Their inclusion is not essential for the discussion at hand. Three possibilities exist for a nonlinear interaction between two harmonics represented by the ordered pair, $\left(n_{1}, n_{2}\right)$ : (i) $n_{1}>n_{2}$, in which case the outcome of backscatter has a positive sign, (ii) $n_{1}<n_{2}$, backscatter is negative in sign, and (iii) $n_{1}=n_{2}$, which represents a self- interaction of a harmonic (or the fundamental), producing outscatter but no backscatter. Outscatter is identical for cases (i) and (ii). Each nonlinear interaction between a given pair $\left(n_{1}, n_{2}\right)$ produces both outscatter and backscatter, with the exception of case 


\section{TABLE II}

Example of Terms Produced by Nonlinear Interaction and Their Simplification (11 Terms Produced in 2nd Iteration of 1st Time Step in Symbolic Computation)

(1) $A^{2} k \sin (k x) \cos (k x)=\frac{1}{2} A^{2} k \sin (2 k x)$
(2) $-2 A^{2} k^{3} t R \sin (k x) \cos (k x)=-A^{2} k^{3} t R \sin (2 k x)$

$\{\mathrm{B}\}$ $\{C\}^{*}$

(3) $-A^{3} k^{2} t \sin (k x) \cos (2 k x)=-\frac{1}{2} A^{3} k^{2} t \sin (3 k x)+\frac{1}{2} A^{3} k^{2} t \sin (k x)$

(4) $-\frac{1}{2} A^{3} k^{2} t \sin (2 k x) \cos (k x)=-\frac{1}{4} A^{3} k^{2} t \sin (3 k x)-\frac{1}{4} A^{3} k^{2} t \sin (k x)$

(5) $\frac{1}{2} A^{3} k^{4} t^{2} R \sin (2 k x) \cos (k x)=\frac{1}{4} A^{3} k^{4} t^{2} R \sin (3 k x)+\frac{1}{4} A^{3} k^{4} t^{2} R \sin (k x)$

(6) $\frac{1}{2} A^{4} k^{3} t^{2} R \sin (2 k x) \cos (2 k x)=\frac{1}{4} A^{4} k^{3} t^{2} R \sin (4 k x)$

(7) $A^{2} k^{5} t^{2} R^{2} \sin (k x) \cos (k x)=\frac{1}{2} A^{2} k^{5} t^{2} R^{2} \sin (2 k x)$

(8) $A^{3} k^{4} t^{2} R \sin (k x) \cos (2 k x)=\frac{1}{2} A^{3} k^{4} t^{2} R \sin (3 k x)-\frac{1}{2} A^{3} k^{4} t^{2} R \sin (k x)$

(9) $A k^{2} R \sin (k x)=$ same

(10) $-A k^{4} t R^{2} \sin (k x)=$ same

(11) $-2 A^{2} k^{3} t R \sin (2 k x)=$ same $\{\mathrm{A}\}$

* Denotes backscatter.

Note. A, B, C, D, and E denote terms with common factors.

(iii), and the resulting amplitudes are identical for outscatter and backscatter, as reflected in the amplitude factor on the RHS of (9),

$$
\text { amplitude factor }=A^{n_{1}+n_{2}+2\left(j_{1}+j_{2}-2\right)} \text {. }
$$

A simple example elucidates the great number of multiplicative combinations that arise in the symbolic computations. If at the beginning of a given time step there are 6 harmonics, plus the fundamental, and each has 4 terms, then there are a total of $4(6+1)=28$ terms representing momentum. Therefore, the momentum multiplied by its derivative yields $28^{2}=784$ quadratic terms at the new time step. Additionally, 28 linear terms are generated from the second order term in the Burgers equation, for a total of 812 terms. Table II displays the 11 resulting terms produced by symbolic computation of the Burgers equation at the second (i.e., final) iteration of the 1st time step. At this point, the total number of terms is quite small compared to subsequent time steps when combinatorial explosion occurs. Also shown in the table are the conversion of quadratic terms through use of a trigonometric identity, as described in Appendix A, and the combination of like terms. Outscatter and backscatter are denoted in the table. The first eight terms shown in the table result from nonlinear interactions and the last three terms stem solely from the linear viscous term of the Burgers equation. An important aspect identified in the table is that the second term, which is a nonlinear inertial term, combines with the last term, a strictly linear term stemming from viscous damping. Thus, the interplay between inertial and viscous terms is revealed. 
The integer exponent of the amplitude factor, (10), embodies complex combinations of harmonic interactions. Signifying the exponent as $E=n_{1}+n_{2}+2\left(j_{1}+j_{2}-2\right)$, several scenarios can be explored. If only the leading terms of an interacting harmonic pair are considered, then $j_{1}=j_{2}=1$, and the exponent reduces to $E=n_{1}+n_{2}$. Various wavenumber pairs, $\left(n_{1}, n_{2}\right)$, potentially comprise $E$. Obviously, the higher the value of $E$ is, the greater the complexity. Except when $n_{1}=n_{2}$, each ordered pair, $\left(n_{1}, n_{2}\right)$, has a counterpart pair, $\left(n_{2}, n_{1}\right)$, with the two elements in reversed order. This is evident in Table II, which shows that the companion pairs exist in separate terms that may or may not have common signs or multipliers. One pair of the set potentially yields an opposite sign in backscatter, which does not in general lead to canceling effects, as seen in the table. If $n_{1}=n_{2}$, no backscatter is produced, as previously explained.

If other than leading terms are considered in the preceding analysis (i.e., $j_{1} \neq 1$ and $j_{2} \neq 1$ ), then the scenarios involving the exponent of the amplitude factor become more complex, but follow a similar line of reasoning. For example, assume $j_{1} \neq 1$ and $j_{2} \neq 1$ in (10). Since $n_{1}+n_{2}=E-2\left(j_{1}+j_{2}-2\right)$, setting $E=14$ and $j_{1}+j_{2}=5$ yields $n_{1}+n_{2}=8$. The companion harmonic pairs (i) $(2,6)$ and $(6,2)$, (ii) $(3,5)$ and $(5,3)$, and (iii) $(4,4)$ satisfy the criterion $n_{1}+n_{2}=8$. But for each of these, the following combinations of term pairs, $\left[j_{1}, j_{2}\right]$, satisfy $j_{1}+j_{2}=5:[1,4],[4,1],[2,3]$, and [3, 2] in which element order in each pair is important. Because there are a total of 5 harmonic pairs, and 4 term pairs for each harmonic pair, a total of 20 interactions satisfy the criterion $E=14$. All of the harmonic pairs produce outscatter to the 7 th harmonic, because $n_{1}+n_{2}=8$, but the harmonic pairs $(2,6)$ and $(6,2)$ produce backscatter to the 3rd harmonic. The pairs $(3,5)$ and $(5,3)$ produce backscatter to the 1 st harmonic, and $(4,4)$ produces no backscatter. It is evident there is a large and complex combination of harmonic interactions at play in the solution to the Burgers equation.

Table III displays combinations of nonlinear harmonic interactions, $\left(n_{1}, n_{2}\right)$, that potentially produce outscatter and backscatter in the fundamental and the 10 harmonics produced in the symbolic solution. However, the companion harmonic pairs denoted by $\left(n_{2}, n_{1}\right)$ are omitted for presentational convenience, but self-interactions denoted by $n_{1}=n_{2}$ are included. Note that outscatter combinations are fewer in number compared to backscatter combinations, and that the fundamental only experiences backscatter. However, solution

TABLE III

Combinations of Harmonic Pairs Producing Outscatter and Backscatter for Fundamental and Various Harmonics

\begin{tabular}{cllllllllllllllll}
\hline $\begin{array}{c}\text { Affected } \\
\text { harmonic }\end{array}$ & \multicolumn{1}{l}{$\begin{array}{c}\text { Outscatter } \\
\text { combinations }\end{array}$} & \multicolumn{1}{c}{$\begin{array}{c}\text { Backscatter } \\
\text { combinations }\end{array}$} \\
\hline Fundamental & None & & $(1,2)$ & $(2,3)$ & $(3,4)$ & $(4,5)$ & $(5,6)$ & $(6,7)$ & $(7,8)$ & $(8,9)$ & $(9,10)$ & $\ldots$ \\
1st harmonic & $(1,1)$ & & $(1,3)$ & $(2,4)$ & $(3,5)$ & $(4,6)$ & $(5,7)$ & $(6,8)$ & $(7,9)$ & $(8,10)$ & $(9,11)$ & $\ldots$ \\
2nd harmonic & $(1,2)$ & & $(1,4)$ & $(2,5)$ & $(3,6)$ & $(4,7)$ & $(5,8)$ & $(6,9)$ & $(7,10)$ & $(8,11)$ & $(9,12)$ & $\ldots$ \\
3rd harmonic & $(1,3)$ & $(2,2)$ & & $(1,5)$ & $(2,6)$ & $(3,7)$ & $(4,8)$ & $(5,9)$ & $(6,10)$ & $(7,11)$ & $(8,12)$ & $\ldots$ \\
4th harmonic & $(1,4)$ & $(2,3)$ & & $(1,6)$ & $(2,7)$ & $(3,8)$ & $(4,9)$ & $(5,10)$ & $(6,11)$ & $(7,12)$ & $(8,13)$ & $\ldots$ \\
5th harmonic & $(1,5)$ & $(2,4)$ & $(3,3)$ & & $(1,7)$ & $(2,8)$ & $(3,9)$ & $(4,10)$ & $(5,11)$ & $(6,12)$ & $(7,13)$ & $\ldots$ \\
6th harmonic & $(1,6)$ & $(2,5)$ & $(3,4)$ & & $(1,8)$ & $(2,9)$ & $(3,10)$ & $(4,11)$ & $(5,12)$ & $(6,13)$ & $(7,14)$ & $\ldots$ \\
7th harmonic & $(1,7)$ & $(2,6)$ & $(3,5)$ & $(4,4)$ & & $(1,9)$ & $(2,10)$ & $(3,11)$ & $(4,12)$ & $(5,13)$ & $(6,14)$ & $\ldots$ \\
8th harmonic & $(1,8)$ & $(2,7)$ & $(3,6)$ & $(4,5)$ & & $(1,10)$ & $(2,11)$ & $(3,12)$ & $(4,13)$ & $(5,14)$ & $(6,15)$ & $\ldots$ \\
9th harmonic & $(1,9)$ & $(2,8)$ & $(3,7)$ & $(4,6)$ & $(5,5)$ & & $(1,11)$ & $(2,12)$ & $(3,13)$ & $(4,14)$ & $(5,15)$ & $\ldots$ \\
10th harmonic & $(1,10)$ & $(2,9)$ & $(3,8)$ & $(4,7)$ & $(5,6)$ & & $(1,12)$ & $(2,13)$ & $(3,14)$ & $(4,15)$ & $(5,16)$ & $\ldots$ \\
\hline
\end{tabular}


results presented in Subsection 3.4 show that most backscatter combinations are of negligible consequence. In contrast, all outscatter combinations are found to be significant, but the strongest effect always involves the fundamental.

The preceding analysis provides a framework to analyze and interpret the results produced by the symbolic computation of the Burgers equation. Referring to (8), each of the $J$ inviscid and $J$ viscous terms comprising the amplitude, $F_{n}$, of each harmonic in the symbolic solution is itself a composite which embodies outscatter, backscatter, and viscous damping that result from potentially large numbers of interacting harmonic pairs. It is instructive to examine individual effects that lead to each composite term in $F_{n}$ by investigating intermediate symbolic operations during each time step, similarly to what was described for Table II. Such a task is useful, in general, for identifying and studying various nonlinear inertial interactions, linear damping mechanisms, and the interplay between inertia and viscous damping. However, experience indicates that it would be inefficient, if not intractable, to use the intermediate symbolic operations if the goal is to isolate and assess the integrated individual effects of outscatter, backscatter, and viscous damping on each individual harmonic. Therefore, an efficient, supplemental method is developed in Appendix B to achieve the desired result of isolating and separately evaluating the effects of these three key mechanisms.

\subsection{Detailed symbolic results: Analysis of outscatter, backscatter, and viscous damping.} In viewing the symbolic results embodied in Tables I and II, it is useful to explore the fundamental and various harmonics separately. At each time step, the symbolic solution, which is in the form expressed by (8), is post-processed using the method presented in Appendix B in which the following numerical upper limits are applied: $A=0.3$, and $\Delta t=0.55$. The process of determining appropriate numerical values is described in Subsection 3.5.

Figures 5-8 show the evolution of the fundamental and the 1st, 3rd, and 10th harmonics over the seven computed time steps. The fundamental and specific harmonics were chosen to enable a broad analysis and interpretation of the fundamental results of the symbolic solution. The symbols $O_{n_{i}-n_{j}}$ and $B_{n_{i}-n_{j}}$ are employed in the figures to represent the outscatter (i.e., production) and backscatter, and the specific harmonic pairs leading to each effect. $O_{n e t}$ and $B_{n e t}$ denote the net effects of outscatter and backscatter. The viscous damping of the fundamental and three harmonics is shown separately on the figures and labeled explicitly. Each figure shows results for (a) $\mathrm{Re}=500$ and (b) $\mathrm{Re}=\infty$. Care must be exercised in comparing Figs. 5-8, because the vertical scales differ between each figure to accommodate the range of magnitudes of the outscatter, backscatter, and damping for each respective harmonic being displayed. For direct comparison between harmonics, the reader is referred to Figs. 4 and 9 in which all harmonics are displayed on the same vertical scale.

Figure 5 displays solution results for the fundamental. The greatest backscatter, denoted by $B_{1-2}$, stems from the nonlinear interaction between the 1 st harmonic and the fundamental itself. Secondary backscatter occurs through interactions between the 1st and 2nd harmonics, $B_{2-3}$, and between the 2 nd and 3rd harmonics, $B_{3-4}$. Backscatter also results from interactions between the 3rd and 4th harmonics and other higher order pairs differing by $\kappa$ in wavenumber, but those interactions are negligible and not shown on the figure. When $\operatorname{Re}=500$, viscous damping exceeds the primary backscatter in magnitude up until the 2nd time step, beyond which the backscatter increases significantly in magnitude due to the rapid growth of the 1 st harmonic. The effect of damping slowly decreases with each time step as the amplitude of the fundamental decreases, but always exceeds the secondary backscatter. 
(a) $\operatorname{Re}=\mathbf{5 0 0}$

Fundamental

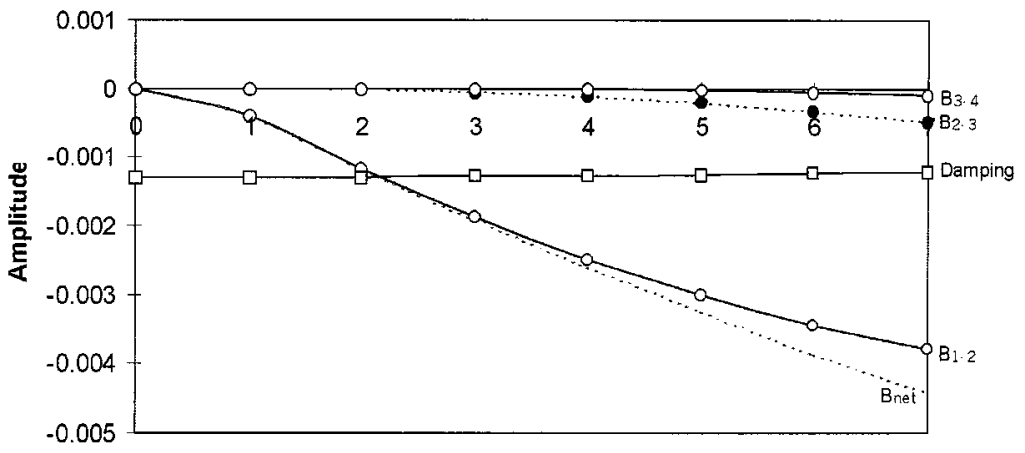

Time Step

(b) $\mathrm{Re}=$ Infinite

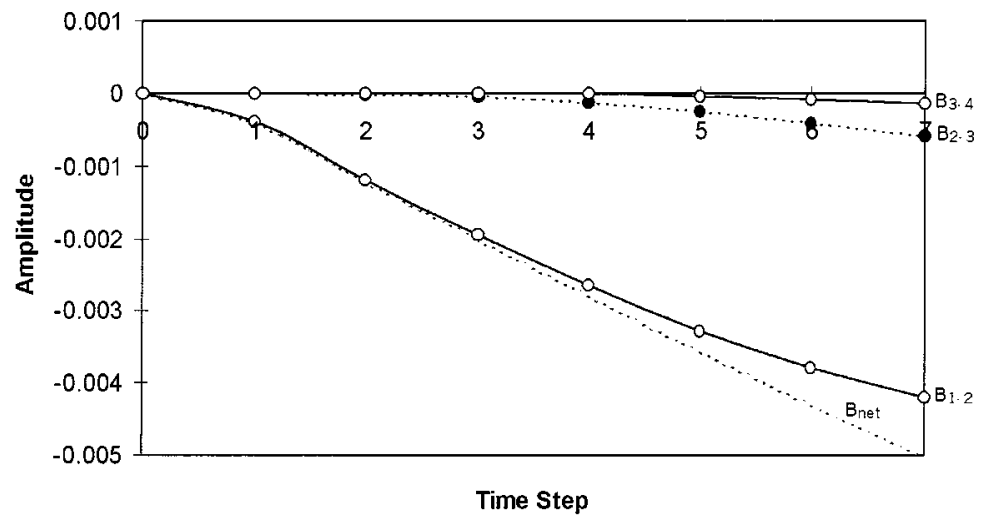

FIG. 5. The time evolution of the fundamental at each of seven discrete time steps in symbolic computation of the Burgers equation for (a) $\mathrm{Re}=500$ and (b) infinite Re. Displays backscatter, $\mathrm{B}_{\mathrm{n} 1-\mathrm{n} 2}$, resulting from interactions between specific harmonic pairs, $\left(n_{1}, n_{2}\right)$, and damping due to viscosity. Example $B_{2-3}$ represents backscatter due to interaction between 1 st and 2 nd harmonics.

In all cases, both damping and backscatter diminish the magnitude of the fundamental. There is no outscatter to the fundamental due to the absence of lower order harmonics.

When $\operatorname{Re}=\infty$, there is no damping, but by comparing parts (a) and (b) of Fig. 5, it is apparent that the effects of backscatter increase by only a small amount. Thus, viscosity does not have a large influence on the fundamental over the seven time steps computed.

The 1st harmonic reveals more intricate behavior. Time histories of the production (i.e., outscatter), backscatter, and viscous damping are displayed in Fig. 6. The 1st harmonic is created at the 1 st time step by self-interaction of the fundamental, as denoted by $O_{1-1}$ in the figure, and continues to grow with time, but at a decreasing rate as the fundamental diminishes. Being an odd harmonic, its amplitude is negative. Its only mode of production is through the fundamental, but it experiences backscatter due to interaction between the fundamental and 2 nd harmonic, noted by $B_{1-3}$ in the figure, and between the 1 st and 3rd harmonics, shown as $B_{2-4}$. Other modes of backscatter are negligible and not shown. Production swamps backscatter and damping over all seven time steps. Backscatter and 
(a) $\operatorname{Re}=\mathbf{5 0 0}$

1st Harmonic

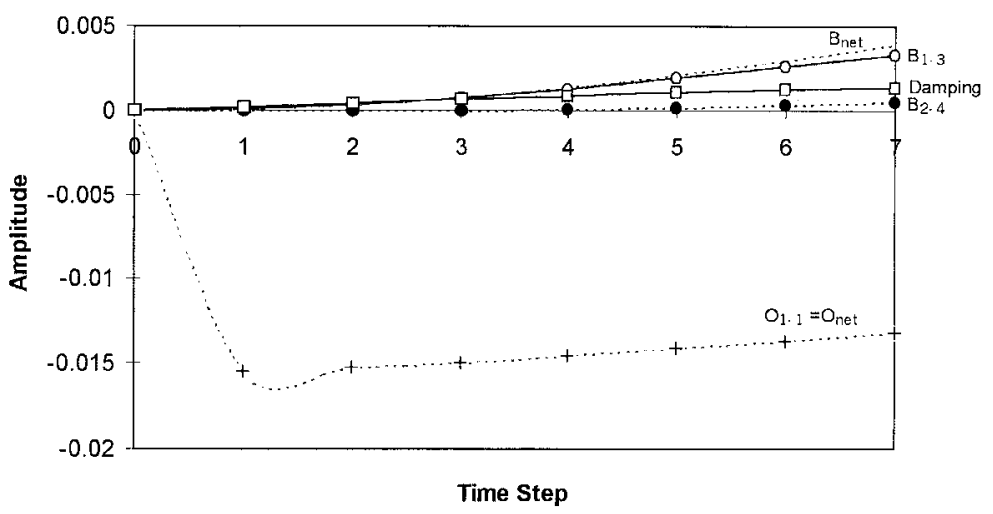

(b) $\mathrm{Re}=$ Infinite

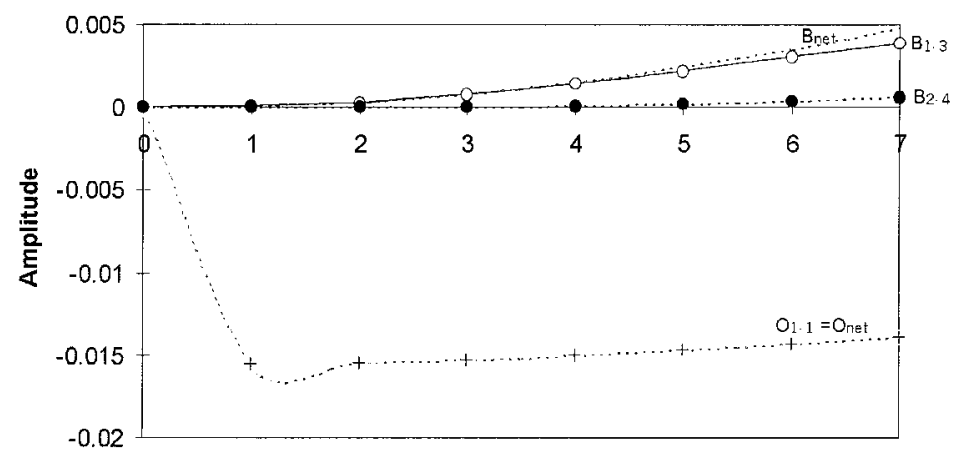

Time Step

FIG. 6. The time evolution of the 1 st harmonic. Displays backscatter, $\mathrm{B}_{\mathrm{n} 1-\mathrm{n} 2}$, and outscatter, $\mathrm{O}_{\mathrm{m} 1-\mathrm{m} 2}$, resulting from interactions between specific harmonic pairs, $\left(\mathrm{n}_{1}, \mathrm{n}_{2}\right)$ and $\left(\mathrm{m}_{1}, \mathrm{~m}_{2}\right)$, and damping due to viscosity. Example $\mathrm{B}_{1-3}$ represents backscatter due to interaction between fundamental and 2 nd harmonic.

damping serve to diminish the amplitude of the 1st harmonic, and damping increases with time because the harmonic is growing.

When $\operatorname{Re}=\infty$, there is no damping, but production and backscatter increase by only a small amount, as shown in Fig. 8. Like the fundamental, the 1st harmonic is affected more by inviscid mechanisms than by viscosity throughout the time evolution of the symbolic computation.

Bypassing the 2nd harmonic, an analysis is made of the 3rd harmonic due to its greater complexity. While the 2 nd harmonic is created solely by interactions between the fundamental and the 1 st harmonic, the 3rd harmonic is created from outscatter produced by two distinct pairs of interactions: (a) the fundamental and the 2nd harmonic, and (b) the selfinteractions of the 1 st harmonic. The two production mechanisms are represented by $O_{1-3}$ and $O_{2-2}$ in Fig. 7, in which $O_{1-3}$ is nearly three times as large as $O_{2-2}$ for all seven time steps. It may seem counterintuitive that $O_{1-3}$ should be that much greater than $O_{2-2}$, given that the 1 st harmonic is straddled by an interaction that produces a greater effect than its own self-interaction. The explanation lies in the large amplitude, $F_{1}$, of the fundamental, such that $\left|F_{1} F_{3}\right|>\frac{1}{2} F_{2}^{2}$, (see discussion in Appendix B). 
(a) $R=500$

Ird Hermonic

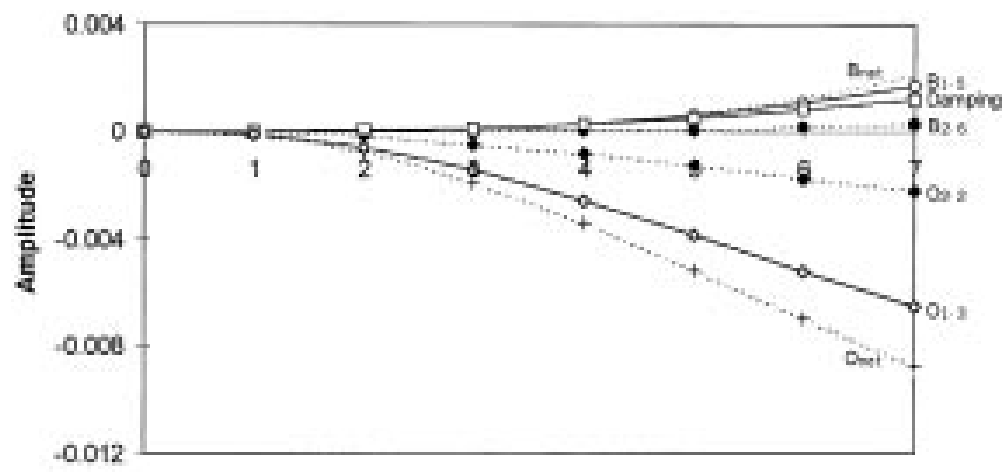

Time Step

(b) Re = Infinite

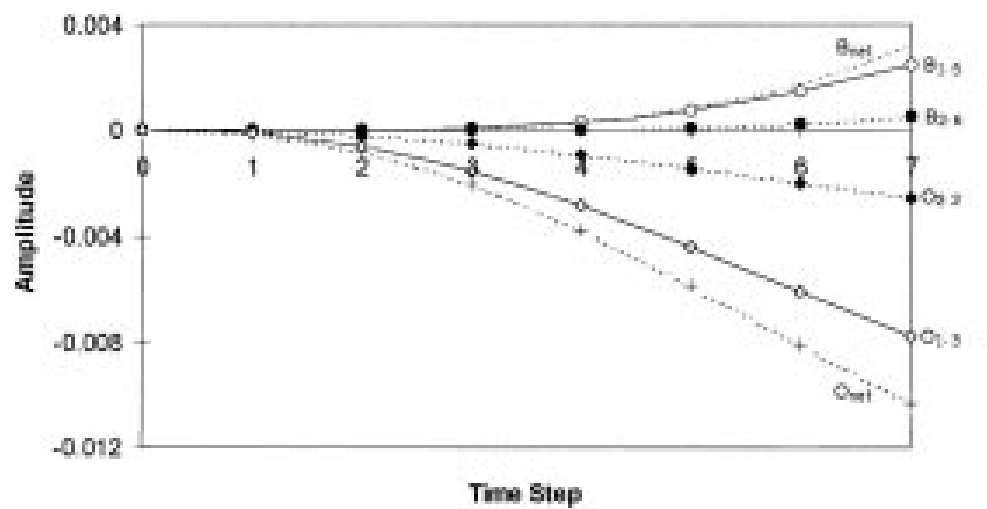

FIG. 7. The time evolution of the 3rd harmonic. Displays outscatter, backscatter, and viscous damping.

Backscatter to the 3rd harmonic occurs due to interaction between the fundamental and 4 th harmonic and between the 1 st and 5 th harmonics, as shown in Fig. 7 by $B_{1-5}$ and $B_{2-6}$, in which $B_{1-5} \gg B_{2-6}$. All higher order backscatter is negligible and therefore excluded. The net effect of backscatter is about one-fourth that of production, but damping is comparable in magnitude to primary backscatter, so the net effect of backscatter and damping is quite significant on the 3rd harmonic.

Lack of damping at $\mathrm{Re}=\infty$ has a more significant effect on the 3rd harmonic than for the lower order harmonics and the fundamental, as shown in Fig. 7. Beginning with the 3rd harmonic, the solution revealed that the lack of viscosity has an increasingly profound effect on both production and backscatter with increasing harmonic order, with the strongest impact being on backscatter. Results for higher order harmonics are not displayed.

Analysis also reveals that for each successively higher order harmonic, the role of damping increases to the point of exceeding the effect of backscatter, and the combination of damping and backscatter becomes increasingly significant relative to the net effect of production. This is certainly an intuitive result. The ratio of the magnitude of production to the combined magnitude of damping and backscatter, however, is about three for the 8th harmonic. Thus, production, or outscatter, continues to play the dominant role. 
(a) $\operatorname{Re}=\mathbf{5 0 0}$

10th Harmonic

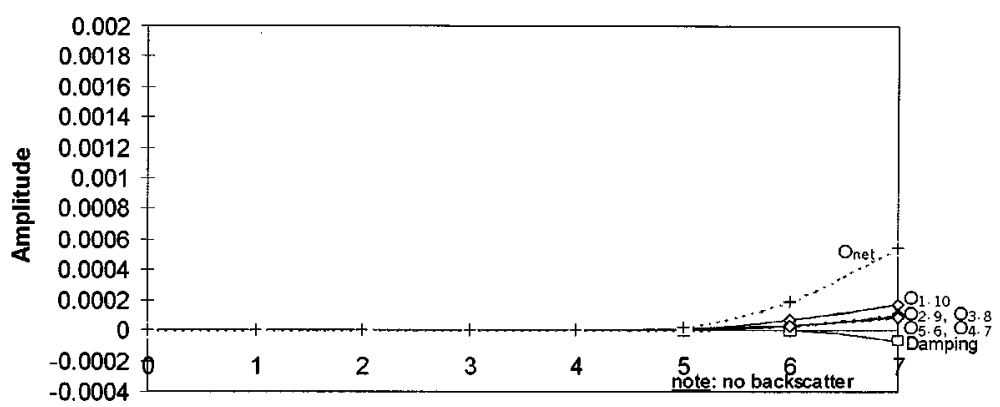

Time Step

\section{(b) $R e=$ Infinite}

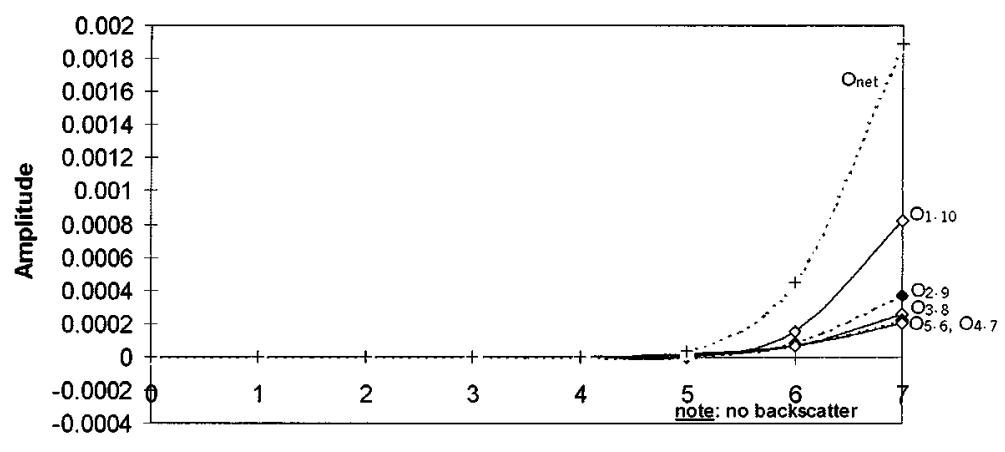

Time Step

FIG. 8. The time evolution of the 10th harmonic. Displays outscatter and viscous damping. Being the highest order harmonic in the symbolic solution, no backscatter is present.

More modes of production are present with increasing harmonic order, as evident in the figures and in Table III, due to the greater number of associated lower order harmonics. Thus, while the 5th harmonic has three production modes, the 7 th harmonic has four. By the 9th and 10th harmonics there are five modes of production, as shown in Fig. 8 for the 10th harmonic. The strongest, or primary, mode of production was found to always involve the fundamental. Each individual secondary mode of production is smaller in magnitude than the primary mode. With increasing harmonic order, however, there are more secondary modes and collectively they can produce a greater effect than the primary mode of production. It is therefore apparent that as time evolves and additional higher order harmonics are produced, the mechanisms of outscatter become increasingly numerous and complex. On the other hand, there are at most only two significant backscatter combinations for all the harmonics produced in the symbolic solution, and only one of those has a primary effect. The 9th harmonic has only one backscatter mode and the 10th harmonic has none, as will be discussed subsequently.

Three key facts emerge regarding the mechanisms and behavior of backscatter in the symbolic solution of the Burgers equation. First of all, significant backscatter to each harmonic except the 1 st results solely from interactions between a harmonic pair containing a lower order harmonic and a higher order harmonic relative to the harmonic being influenced. 
(a) $\operatorname{Re}=500$

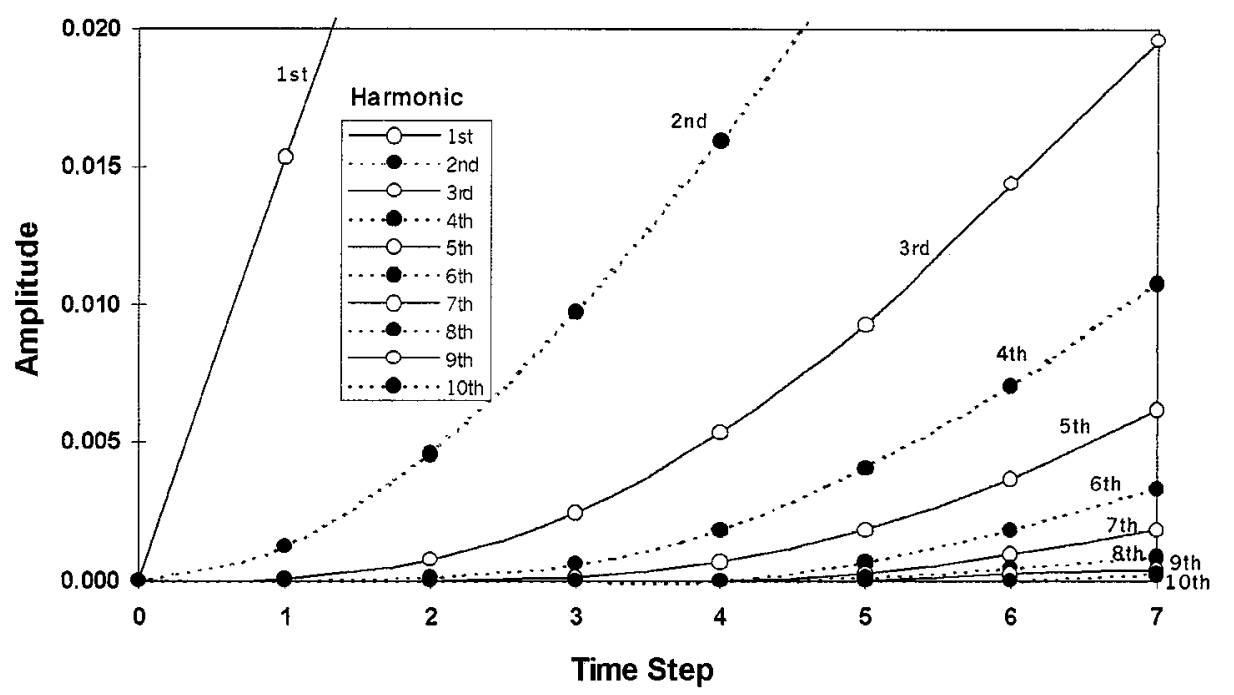

(b) $\mathbf{R e}=$ Infinite

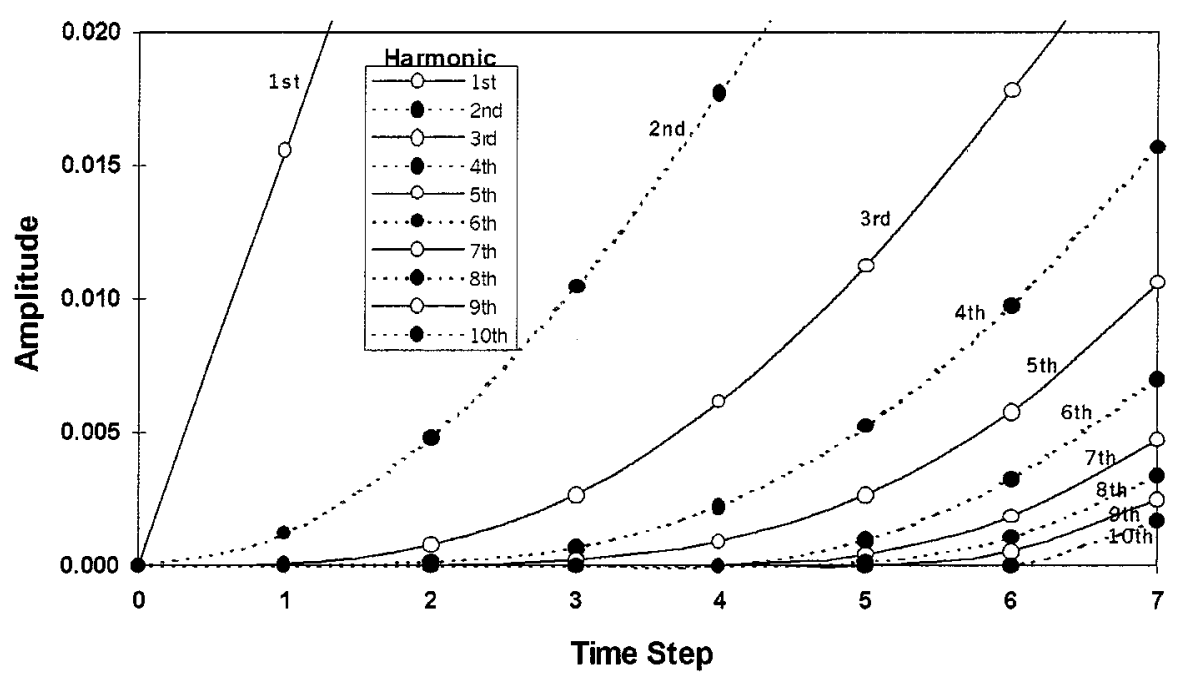

FIG. 9. The comparative amplitudes of the fundamental and 1st through 10th harmonics at each of seven discrete time steps in symbolic computation of the Burgers equation for (a) $\operatorname{Re}=500$ and (b) infinite Re. The slope of each curve reveals the time rate of change of each harmonic.

In all cases observed, the fundamental interacting with the adjacent higher order harmonic creates the dominant backscatter effect. Thus backscatter cannot be characterized as a "localized" nonlinear effect. Backscatter due to interactions in which both harmonics in the pair are of higher order than the affected harmonic was found to be negligible in the preliminary computations. This certainly may not be the case for solutions with initial conditions that contain high order harmonics of large amplitude. There may be other cases where the cumulative backscatter created by a myriad of high order pairs could be significant. Second, the effect of backscatter is to deplete momentum in all cases, regardless of the value of Re. 
Thus, viscous damping and backscatter work in concert to decrease the momentum. For time evolution beyond the current preliminary results, there may be cases where the effect of backscatter is to supply momentum to lower order harmonics and the fundamental. Third, as the symbolic solution evolves, the highest order harmonic included at any given time step experiences production but no backscatter because of the absence of the next higher order harmonic. This is illustrated in Fig. 8 for the 10th harmonic, the highest order considered in the solution.

It is argued that the unavoidable exclusion of backscatter for the highest order harmonic is of negligible consequence to the symbolic solution. First of all, by virtue of the filtering process described in Subsection 3.5, the amplitude of the highest order harmonic included in the solution at each time step is inherently small. Each successive higher order harmonic is allowed into the solution only when production (i.e., outscatter) causes the current highest order harmonic to exceed a certain magnitude relative to its lower order neighbor. The two primary ways the highest order harmonic affects the solution is through backscatter to its lower order neighbor and production to the next higher order harmonic. As was described in the preceding discussion, backscatter depletes momentum, at least for the preliminary computations undertaken in this study. Thus, the lack of backscatter to the highest order harmonic serves to make its amplitude somewhat larger than it otherwise would be. Through backscatter, it therefore causes its lower order neighbor to be somewhat smaller than otherwise. Then, because production of the highest order harmonic is directly related to the magnitude of its lower order neighbor, it in turn receives less production and grows less in amplitude. So the tendency of the highest order harmonic to become too large due to its not receiving backscatter is counteracted by a self-adjusting process. A more rigorous validation of solution accuracy, with truncation of higher order harmonics, is that the kinetic energy of the symbolic solution of the inviscid Burgers equation (i.e., $\operatorname{Re}=\infty$ ) was found to remain constant to within $0.14 \%$ as the solution progressed in time. Thus energy is conserved to a high order of accuracy.

Figure 9 displays the "birth" and growth of the 10 harmonics produced by the solution. The fundamental is excluded from the figure. The 4th harmonic is "born" at the 3rd time step and its growth accelerates in time. The 7th harmonic is born at the 4th time step when $\operatorname{Re}=\infty$, and at the 5th time step when $\operatorname{Re}=500$. The figure clearly reveals the enhanced effect of viscous damping with increasing harmonic order.

3.5. Filtering negligible symbolic terms. As previously discussed, the symbolic results displayed in Tables I and II represent a filtered set of computations. Sensitivity tests were done in an attempt to develop an effective process to identify and eliminate negligible terms prior to symbolic multiplication in order to prevent or minimize unproductive combinatorial explosion and maintain solution accuracy. However, a significant portion of the filtering process can only be performed after symbolic operations have produced a myriad of both critical and superfluous terms. Filtering is based primarily on evaluating the symbolic terms comprising the amplitudes of the fundamental and each harmonic. The separate issue of the addition, or "birth," of higher order harmonics into the symbolic solution was described in Subsection 3.4 .

In (8) it is apparent that the leading inviscid and viscous terms for each harmonic are $C_{n_{1}} A^{n} \kappa^{n-1} \Delta t^{n-1}$ and $D_{n_{1}} A^{n} \kappa^{n+1} \Delta t^{n} \frac{1}{\mathrm{Re}}$, in which $C_{n_{1}}$ and $D_{n_{1}}$ are their leading coefficients. The second inviscid and viscous terms are $C_{n_{2}} A^{n+2} \kappa^{n+1} \Delta t^{n+1}$ and $D_{n_{2}} A^{n+2} \kappa^{n+3} \Delta t^{n+2} \frac{1}{\mathrm{Re}}$, with leading coefficients $C_{n_{2}}$ and $D_{n_{2}}$. The relationship is identical for all successive terms 
considered. In evaluating the magnitude of terms at each time step, all terms are compared by ratio to their respective lead term (i.e., inviscid and viscous terms are treated separately). The exception is the fundamental for which the lead term is $A$, the initial amplitude. Thus all terms for the fundamental are compared instead to the 2nd inviscid term, $C_{1_{2}} A^{3} \kappa^{2} \Delta t^{2}$. For all harmonics, the resulting ratios, Ratio $_{I}$ and Ratio ${ }_{V}$, are as follows for the inviscid and viscous terms, respectively,

$$
\operatorname{Ratio}_{I}=\frac{C_{n_{j}}}{C_{n_{1}}}[A \kappa \Delta t]^{2 j-2}
$$

and

$$
\text { Ratio }_{V}=\frac{D_{n_{j}}}{D_{n_{1}}}[A \kappa \Delta t]^{2 j-2}
$$

with $j=2,3,4, \ldots, J$. As will be discussed, $A \kappa \Delta t \ll 1$. This composite factor, which appears in brackets in (11a) and (11b), is raised to an additional power of two for each successive higher order term being compared to the leading term. The magnitudes of the coefficient ratios $C_{n_{j}} / C_{n_{1}}$ and $D_{n_{j}} / D_{n_{1}}$ in (11a) and (11b) play a definitive role in discerning whether a higher order term is negligible and can be excluded from the symbolic solution at any given time step.

The filtering process implicitly requires upper numerical limits on $A$ and $\Delta t$, and a lower limit on $\mathrm{Re}$ in order to evaluate the relative magnitudes of higher order symbolic terms and to establish the range of numerical parameters for which the symbolic solution applies. Higher order terms were eliminated if it was determined that their absence would not compromise the cumulative accuracy of subsequent symbolic operations. Sensitivity tests indicate that setting $\operatorname{Ratio}_{I}=$ Ratio $_{V} \approx 0.01$ yields an effective filtering criterion. Given this criterion, appropriate upper numerical limits were determined for the initial amplitude, $A$, and the non-dimensional time step, $\Delta t$, as $A \leq 0.3$ and $\Delta t \leq 0.055$. Because the nondimensional wavenumber is large, i.e., $\kappa=2 \pi$, the preceding limits on $\Delta t$ and $A$ assure that $A \kappa \Delta t \ll 1$, which reduces the magnitude of higher order terms and enables their truncation without compromising the validity of the symbolic solution. In fact, $A \kappa \Delta t \approx 0.1$, such that any combination of $A$ and $\Delta t$ that satisfies $A \Delta t \approx 0.1 / 2 \pi$ provides similar results when substituted into the symbolic solution. That is, a larger (smaller) $A$ accompanies a smaller (larger) $\Delta t$, but the character of the generated waveform and the relationship between the fundamental and harmonics remain similar. If one were to seek some combination of $A$ and $\Delta t$ which yielded $A \kappa \Delta t>0.1$, it would be necessary to retain a greater number of higher order terms as compensation. The goal is to maximize accuracy with the symbolic solution with a minimum number of terms.

Numerical values of $A, \Delta t$, and Re are not used, of course, in the symbolic computations. However, once the symbolic solution is obtained, appropriate numerical values can be substituted for $\Delta t, A, \operatorname{Re}$, and $x$, within their appropriate ranges, to explore numerical implications of the solution. Note that the spatial variable, $x$, is also non-dimensional and falls in the range $0 \leq x \leq 1$. As mentioned in Subsection 3.2, the symbolic solution of the Burgers equation presents no upper limit to $\mathrm{Re}$, but requires that $\mathrm{Re} \geq 500$. Extending the symbolic solution to lower values of Re would require retention of viscous terms of $O(1 / \mathrm{Re})^{2}$ or higher. Symbolic computation reveals that terms of $O(1 / \mathrm{Re})^{2}$ have the form $E_{n_{j}} A^{n+2 j-2} \kappa^{n+2 j+1} \Delta t^{n+2 j-1}(1 / \mathrm{Re})^{2}$, yielding a ratio of $\left(E_{n_{j}} / D_{n_{j}}\right) \kappa^{2} \Delta t(1 / \mathrm{Re})$ to terms of $O(1 / \mathrm{Re})$ in (8). To justify the exclusion of the higher order terms, the ratio must be small. 
A reasonable criterion is that $\left(E_{n_{j}} / D_{n_{j}}\right) \kappa^{2} \Delta t(1 / \mathrm{Re}) \approx 0.1$ as an upper limit. Thus, if $E_{n_{j}}$ and $D_{n_{j}}$ were of equal magnitude, the lower limit on Reynolds number would be $\operatorname{Re} \geq 20$. However, the symbolic results indicate that a more typical representative ratio between the leading coefficients is $E_{n_{j}} / D_{n_{j}} \approx 40$, in which case the lower limit on Reynolds number would be $\operatorname{Re} \geq 900$. Sensitivity runs with the symbolic computations, however, showed an effective limit to be $\mathrm{Re} \geq 500$. Highest order harmonics are most sensitive to the exclusion of $O(1 / \mathrm{Re})^{2}$ terms. For longer time evolution these terms, and possibly even higher order ones, must be retained. Therefore, it is clear that efforts exploring low Re flows would require solution refinements. Also, current results suggest that longer time evolution with high values of Re may confront the need to retain $O(1 / \mathrm{Re})^{2}$ terms to maintain solution accuracy, as well. The inclusion of more terms increases the computational complexity and intensifies combinatorial explosion. Thus, we face the irony that while numerical solutions experience instabilities with high Re flows, the symbolic approach has difficulty with low Re but can inherently handle flows with infinite Re. This is fortuitous because turbulent flows at extremely high values of Re are the most elusive and interesting in research and in practical applications.

\section{SUMMARY AND CONCLUSIONS}

A novel approach based on recursive symbolic computation is introduced for the approximate analytic solution of both the inviscid and viscous Burgers equations. Through the recursive process, symbolic representations of momentum are obtained continuously in space at discrete increments in time. Although approximate, the solution can be obtained to arbitrary, high order accuracy. Once obtained, appropriate numerical values can be inserted into the symbolic solution to explore parametric variations.

The symbolic computations allow examination of the solution at stages prior to combining and simplifying terms during each time step. Thus, one can gain a deeper understanding of the precise role and character of all nonlinear interactions, viscous damping, and the interplay between inertial and viscous mechanisms. This is not done easily, if at all, with numerical solutions, which produce only the net effect of nonlinear interactions and other physical processes. Likewise, the ability to readily isolate and assess the integrated individual effects of outscatter, backscatter, and viscous damping by post-processing symbolic results lends power over numerical methods. The same basic arguments apply in comparing symbolic computation with traditional nonlinear analytical solutions. An added feature of the symbolic approach is that "turbulence" manifests as a process of combinatorics, providing a fresh view to a classical problem, even though results are limited to the 1-D Burgers equation. Based on the preliminary efforts presented, it appears that symbolic computation may be quite effective in unveiling the "anatomy" of the myriad inertial and viscous interactions that underlie fundamental turbulent behavior.

Because of the tendency of nonlinear symbolic operations to produce combinatorial explosion, future efforts will require the development of improved filtering processes to select and eliminate negligible high order terms. Thus, while the preliminary results are limited to relatively short time evolution, it is envisioned that future efforts will explore more fully developed flows. With further research, there may be potential to extend the symbolic computations to 2-D and 3-D studies.

The symbolic approach is superior to numerical techniques in four distinct ways: (a) its ability to reveal and elucidate direct nonlinear interactions between waveforms, including 
the interplay between inertia and viscosity; (b) its ability to broadly explore parameter space, most importantly variations in Re, by simple substitution of desired numerical values into the analytical solution; (c) its ability to deal with a wide range of $\operatorname{Re}$ flows $(500 \leq \operatorname{Re} \leq \infty)$; and (d) it produces no false dispersion or aliasing. In contrast, numerical solutions require an additional simulation for every variation in a parameter, and DNS is limited to $\operatorname{Re} \leq 4000$.

Potential applications include the development of improved subgrid scale (SGS) parameterizations for large eddy simulation (LES) models, and studies that complement direct numerical simulation (DNS) in exploring fundamental aspects of turbulent flow behavior. In that regard, the symbolic approach may overcome the low Reynolds number restrictions faced by DNS.

\section{APPENDIX A: QUADRATIC NONLINEAR INTERACTIONS}

Simple symbolic "calculations" with the dimensional term $u \partial u / \partial x$ demonstrate the general character of quadratic nonlinearity as embodied in both the Burgers equation and the Navier-Stokes equations. The initial value $u=A \sin (\kappa x)$ is assumed, where the amplitude, $A<1$, and wavenumber is defined by

$$
\kappa=\frac{2 \pi}{L_{0}},
$$

in which $L_{0}$ is the wavelength for the initial condition and represents the "fundamental" wave spanning a periodic spatial domain. Applying the initial condition to the quadratic nonlinearity yields

$$
u \frac{\partial u}{\partial x}=\kappa A^{2} \sin (\kappa x) \cos (\kappa x)=\frac{1}{2} \kappa A^{2} \sin (2 \kappa x),
$$

in which the trigonometric identity

$$
\sin \theta \cos \phi=\frac{1}{2}[\sin (\theta+\phi)+\sin (\theta-\phi)]
$$

can be used to show that the nonlinearity leads to a doubling of the initial wavenumber. That is, the initial wave, or fundamental, produces a harmonic through the nonlinear process.

It can be shown in general, as implied by (A3), that the quadratic nonlinear interaction between any two waves with respective wavenumbers $\kappa_{1}$ and $\kappa_{2}$ create two additional waves with wavenumbers $\kappa_{1}+\kappa_{2}$ and $\kappa_{1}-\kappa_{2}$ or $-\kappa_{1}+\kappa_{2}$, noting that a sign change can occur through a nonlinear interaction (Minorski [19] can be consulted with respect to quadratic and higher degree nonlinearities.). That is, the nonlinear interaction produces new waves that are larger and smaller than the original interacting waves. This can be summarized as

$$
\begin{gathered}
\kappa_{\text {large }}=\kappa_{1}+\kappa_{2}=\frac{2 \pi}{L_{1} L_{2} /\left(L_{1}+L_{2}\right)} \\
\kappa_{\text {small }}=\kappa_{1}-\kappa_{2}=\frac{2 \pi}{L_{1} L_{2} /\left(L_{1}-L_{2}\right)},
\end{gathered}
$$

where $L_{1}$ and $L_{2}$ correspond to the two interacting waves. Their associated wavelengths are

$$
L_{\text {small }}=\frac{L_{1} L_{2}}{L_{1}+L_{2}}
$$


and

$$
L_{\text {large }}=\frac{L_{1} L_{2}}{L_{1}-L_{2}},
$$

where it is emphasized that the smaller (larger) wavelength is associated with the larger (smaller) wavenumber. The process leading to the creation of smaller waves is referred to as outscatter. Similarly, the creation of larger waves from the nonlinear interaction between smaller waves is termed backscatter. The two nonlinear mechanisms constitute the inertial transfer of momentum between scales. In general, outscatter can be thought of as a production mechanism and backscatter as a modification to existing lower harmonics. However, because both processes constitute the inertial transfer of momentum (and energy) between scales, neither outscatter nor backscatter add to or subtract momentum (or energy) from the system. Thus, use of the word production here only refers to the creation of higher order harmonics at the expense of lower order ones. Actual production to a system is through large-scale shear or buoyancy, which are excluded in the current study.

Beyond the initial interaction of two waves in a time varying nonlinear process, the "birth" of new waves creates a large, increasing number of nonlinear interactions as time evolves. Symbolic computation provides an effective tool to explore the intricacies of such a large number of nonlinear interactions in a study of developing 1-D "burgulence."

\section{APPENDIX B: ISOLATING OUTSCATTER, BACKSCATTER, AND VISCOUS DAMPING}

In this appendix, a method is developed to isolate and integrate the individual effects of outscatter, backscatter, and viscous damping at each discrete time step in the symbolic solution of Burgers equation. The method is applied to the solution in a post-processing fashion.

The solution at each discrete time level can be expressed as

$$
u(x, m \Delta t)=F_{1} \sin (\kappa x)+F_{2} \sin (2 \kappa x)+F_{3} \sin (3 \kappa x)+\cdots+F_{N} \sin (N \kappa x),
$$

in which all terms associated with the amplitude of each respective harmonic are lumped into the symbols $F_{1}, F_{2}, F_{3}$, etc., rather than kept in expanded form. Symbolic integration to the next time level is achieved by substituting (B1) into the forward-in-time algorithm, (7a), to yield

$$
u(x,(m+1) \Delta t)=u(x, m \Delta t)+\kappa \Delta t \sum_{n=1}^{N} \frac{n}{2}\left\{\begin{array}{c}
\text { outscatter } \\
-\left(\sum_{l=1}^{L_{p}} F_{l} F_{|l-n|}-\frac{1}{2} F_{n / 2}^{2}\right) \\
\text { backscatter } \\
+\left(\sum_{l=1}^{L_{b}} F_{l} F_{l+n}\right) \\
\text { viscous damping } \\
-\left(2 n \frac{\kappa}{\operatorname{Re}} F_{n}\right)
\end{array}\right\} \sin (n \kappa x),
$$

in which $n=1,2,3, \ldots, N$ corresponds to the individual harmonics, and the subscript $l=1,2,3, \ldots, L_{p}$ or $L_{b}$ represents the amplitude pairs involved in outscatter or backscatter for each harmonic of the solution. In general $L_{p} \neq L_{b}$, and $L_{p}$ and $L_{b}$ differ from one harmonic to the next. The outscatter, backscatter, and damping terms have been identified in (B2). The term $\frac{1}{2} F_{n / 2}^{2}$ is applied only to production by outscatter of odd harmonics 
(i.e., when $n$ is even) and is excluded for even harmonics (when $n$ is odd). The individual mechanisms for each harmonic are separated as

$$
\begin{aligned}
\text { outscatter } & =-\kappa \Delta t \frac{n}{2}\left(\sum_{l=1}^{L_{p}} F_{l} F_{|l-n|}-\frac{1}{2}\left(F_{n / 2}^{2}\right)_{n=\mathrm{even}}\right), \\
\text { backscatter } & =\kappa \Delta t \frac{n}{2}\left(\sum_{l=1}^{L_{b}} F_{l} F_{l+n}\right),
\end{aligned}
$$

and

$$
\text { viscous damping }=-\kappa^{2} \Delta t n^{2} \frac{1}{\operatorname{Re}} F_{n} .
$$

The expressions (B3), (B4), and (B5) reveal the specific pairs of interacting harmonics that modified a given harmonic in achieving its amplitude at the current discrete time level. By way of example, if we consider the 3rd harmonic, then $n=4$ and production due to outscatter from lower order harmonics is $-2 \kappa \Delta t\left\{F_{1} F_{3}+\frac{1}{2} F_{2}^{2}\right\}$, and the backscatter from higher order harmonics is $2 \kappa \Delta t\left\{F_{1} F_{5}+F_{2} F_{6}+F_{3} F_{7}+\cdots\right\}$. Note that $L_{p}=2$ because only the harmonic pairs $(1,3),(3,1)$, and $(2,2)$ can produce the 3 rd harmonic, whereas the value of $L_{b}$ is theoretically unlimited because any two harmonics that differ in wavenumber by $4 \kappa x$ interact to yield backscatter (see Table III). However, only a small number of backscatter interactions have magnitudes large enough to have a significant effect. Viscous damping at each time step for the $3 \mathrm{rd}$ harmonic is $-16 \kappa^{2} \Delta t \frac{1}{\mathrm{Re}} F_{4}$. To quantify the outscatter, backscatter, and damping, numerical values for $A, \Delta t$, and Re are inserted into each term comprising the amplitudes $F_{1}, F_{2}, F_{3}$, etc. Best accuracy is achieved by averaging the numerical values of $F$ for the preceding and current time levels. In closing this discussion, it is important to emphasize that (B2) is not used to compute the symbolic solution to the Burgers equation. Instead, it is used solely to develop the algorithms necessary to postprocess the symbolic solution in separating and analyzing the integrated effects of outscatter, backscatter, and viscous damping for each time step.

\section{ACKNOWLEDGMENTS}

This project was supported, in part, by the Nelson Research Awards funds of the South Dakota School of Mines and Technology (SDSM\&T) Foundation. Additional support was provided by the Department of Defense Army Research Office under Contract DAAH04-94-G-0420. Dick Farley of the SDSM\&T provided an extremely helpful and detailed technical review of an earlier version of the manuscript. His efforts are greatly appreciated.

\section{REFERENCES}

1. M. Avellaneda, R. Ryan, and W. E, PDFs for velocity and velocity gradients in Burgers' turbulence, Phys. Fluids 7, 3067 (1995).

2. H. Bateman, Some recent researches on the motion of fluids, Mon. Wea. Rev. 43, 163 (1915).

3. D. T. Blackstock, Convergence of the Keck-Boyer perturbation solution for plane waves of finite amplitude in a viscous fluid, J. Acoust. Soc. Am. 39, 411 (1966).

4. J. P. Bouchaud, M. Mezard, and G. Parisi, Scaling and intermittency in Burgers turbulence, Phys. Rev. E 52, 3656 (1995).

5. J. M. Burgers, A mathematical model illustrating the theory of turbulence, Adv. Appl. Mech. 1, 171 (1948).

6. J. M. Burgers, The Nonlinear Diffusion Equation: Asymptotic Solutions and Statistical Problems (Reidel, Boston, 1974), p. 173. 
7. A. Chekhlov and V. Yakhot, Kolmogorov turbulence in a random-force-driven Burgers equation: Anomalous scaling and probability density functions, Phys. Rev. E 52, 5681 (1995).

8. S. Chen and R. H. Kraichnan, Simulations of a randomly advected passive scalar field, Phys. Fluids 10, 2867 (1998).

9. J. D. Cole, On a quasilinear perturbation equation occurring in aerodynamics, Quart. Appl. Math. 9, 225 (1951).

10. R. G. Derickson, Finite Difference Methods for Minimizing False Wave Dispersion in Geophysical Flow Simulations, Ph.D. dissertation, Colorado State University, Fort Collins, Co, 1992, p. 320.

11. W. E, K. Khanin, A. Mazel, and Y. Sinai, Probability distribution functions for the random forced Burgers equation, Phys. Rev. Lett. 78, 1904 (1997).

12. M. J. Feigenbaum, Quantitative universality for a class of nonlinear transformations, J. Stat. Phys. 19, 29 (1978)

13. C. A. J. Fletcher, Burgers equation: a model for all reasons, in Numerical Solution of Partial Differential Equations, edited by J. Noye (North-Holland, Amsterdam, 1982), p. 647.

14. C. A. J. Fletcher, Computational Galerkin Methods (Springer-Verlag, New York, 1984), p. 309.

15. T. Gotoh and R. H. Kraichnan, Steady-state Burgers turbulence with large-scale forcing, Phys. Fluids 10, 2859 (1998).

16. S. N. Gurbatov, S. I. Simdyankin, E. Aurell, U. Frisch, and G. Toth, On the decay of Burgers turbulence, J. Fluid Mech. 344, 339 (1997).

17. E. Hopf, The partial differential equation $u_{t}+u u_{x}=\mu u_{x x}$, Comm. Pure Appl. Math. 3, 201 (1950).

18. M. J. Lighthill, Viscosity effects in sound waves of finite amplitude, in Surveys in Mechanics, edited by G. K. Batchelor, and R. M. Davies (Cambridge Univ. Press, Cambridge, UK, 1956), p. 475.

19. N. Minorski, Introduction to Non-linear Mechanics: Topological Methods, Analytical Methods, Non-linear Resonance, Relaxation Oscillations (Edwards, Ann Arbor, 1947), p. 464.

20. D. Redfern, The Maple Handbook (Springer-Verlag, New York, 1996), p. 495.

21. T. C. Scott, R. A. Moore, M. B. Monahan, and G. J. Fee, Perturbative solutions of quantum mechanical problems by symbolic computation, J. Comput. Phys. 87, 366 (1990).

22. G. B. Whitham, Linear and Nonlinear Waves (Wiley, New York, 1974), p. 636. 